\title{
A linear stability analysis on the onset of thermal convection of a fluid with strongly temperature-dependent viscosity in a spherical shell
}

Received: 2 September 2010 / Accepted: 3 December 2011 / Published online: 29 December 2011

(C) The Author(s) 2011. This article is published with open access at Springerlink.com

\begin{abstract}
A linear stability analysis was performed in order to study the onset of thermal convection in the presence of a strong viscosity variation, with a special emphasis on the condition for the stagnant-lid (ST) convection where a convection takes place only in a sublayer beneath a highly viscous lid of cold fluid. We consider the temporal evolution (growth or decay) of an infinitesimal perturbation superimposed to a Boussinesq fluid with an infinite Prandtl number which is in a static (motionless) and conductive state in a basally heated planar layer or spherical shell. The viscosity of the fluid is assumed to be exponentially dependent on temperature. The linearized equations for conservations of mass, momentum, and internal (thermal) energy are numerically solved for the critical Rayleigh number, $R a_{c}$, as well as the radial profiles of eigenfunctions for infinitesimal perturbations. The above calculations are repeatedly carried out by systematically varying (i) the magnitude of the temperature dependence of viscosity, $E$, and (ii) the ratio of the inner and outer radii of the spherical shell, $\gamma$. A careful analysis of the vertical structure of incipient flows demonstrated that the dominance of the ST convection can be quantitatively identified by the vertical profile of $\Delta_{h}$ (a measure of conversion between horizontal and vertical flows), regardless of the model geometries. We also found that, in the spherical shell relevant to the Earth's mantle $(\gamma=0.55)$, the transition into ST convection takes place at the viscosity contrast across the layer $r_{\eta} \simeq 10^{4}$. Taken together with the fact that the threshold value of $r_{\eta}$ falls in the range of $r_{\eta}$ for a so-called sluggish-lid convection, our finding suggests that the ST-mode of convection with horizontally elongated convection cells is likely to arise in the Earth's mantle solely from the temperature-dependent viscosity.
\end{abstract}

Keywords Mantle convection · Temperature-dependent viscosity · Spherical shell · Linear stability analysis . Stagnant-lid convection

\section{Introduction}

Temperature-dependent viscosity is one of the most important properties that control the convective flow patterns in the mantle of terrestrial planets $[4,26]$. For example, the viscosity varies by several to ten orders of magnitude associated with the temperature change within the Earth's mantle [9,25]. Owing to the strongly temperature-dependent viscosity of mantle materials, a highly viscous lid of cold rocks is expected to develop along the top surface of the mantle, which is quite analogous to the plates or lithospheres of the planetary surfaces $[3,9,10,15,16$, for example]. From this viewpoint, thermal convection of the fluid with strongly temperaturedependent viscosity has attracted much interest, both experimentally and numerically, from researchers on mantle convection [14, for a review].

Communicated by: Malik.

M. Kameyama $(\varangle) \cdot$ H. Ichikawa $\cdot$ A. Miyauchi

Geodynamics Research Center, Ehime University, Matsuyama 790-8577, Japan

E-mail: kameyama@sci.ehime-u.ac.jp 
The influence of the temperature-dependent viscosity on the pattern of the thermal convection has been investigated mainly by theoretical $[19]$ and numerical $[3,9,10,15,16]$ studies in Cartesian domains. From the changes in vertical flow structures, three convective regimes have been identified depending on the magnitude of temperature dependence of viscosity; the small-viscosity-contrast or whole-layer (WH) regime where convection involves all of the fluid in the vessel, the stagnant-lid (ST) regime where convection occurs only beneath a cold stagnant lid that develops along the surface boundary, and the transitional (TR) regime between the WH and ST regimes. Convection of WH-, TR-, and ST-modes arises when the temperature dependence of viscosity is weak, moderate, and strong, respectively, and the transitions between the three convective regimes are induced by the changes in the stiffness of the cold thermal boundary layer coming from the changes in the magnitude of temperature dependence of viscosity. In particular, a theoretical study by [19] for the onset of convection in a planar layer estimated that the ST-mode of convection takes place when the viscosity contrast across the convecting layer exceeds a threshold value of around $\exp (8) \simeq 3,000$, which has been regarded as the critical point for the classification of the convective regimes.

On the other hand, mainly from the numerical models of three-dimensional vessels, it has also been recognized that the temperature-dependent viscosity affects the horizontal length scales of convection cells $[11,13,20,24,29]$. Besides the three regimes described above in terms of the changes in the vertical structures, another suite of three convective regimes have been separately identified; (a) a regime with narrow convection cells for weak temperature dependence, (b) a regime sometimes called "sluggish-lid" (SL) mode for moderate temperature dependence, which is characterized by wide convection cells, and (c) a regime with narrow convection cells for strong temperature dependence. Taken together with the two suites of changes in convective flow regimes, it has often been conjectured that the convection with strongly temperature-dependent viscosity is always characterized by narrow convection cells beneath stagnant lids of cold and highly viscous fluid. Moreover, in almost all of the recent numerical models of convection in three-dimensional spherical shells $[18,27,28]$, the reduction in the horizontal length scales of convection cells has been used as the criterion of the transition into the ST regimes, even though in some of these studies $[18,28]$, the relations between the two series of changes were slightly discussed. Implicit in the above conjecture is the assumption that the two series of changes in convective flow regimes are identical. However, as has been demonstrated from a systematic numerical study for thermal convection with strongly temperature-dependent viscosity in a two-dimensional rectangular box [3], the changes in horizontal and vertical flow structures belong to different series of transitions for convection. In addition, recent numerical studies by using two-dimensional cylindrical $[5,7]$ and three-dimensional spherical shell geometries [17] suggested the existence of convection patterns with large horizontal length scales beneath thick cold thermal boundary layers. It is, therefore, very unlikely that, in a three-dimensional spherical shell geometry, the transition into the ST regime always coincides with the changes from wide to narrow convection cells. This indicates that in order to precisely classify the convective regimes in a spherical shell geometry, the transition into the ST-mode of convection must be identified not indirectly from the horizontal but directly from the vertical flow structures.

In this study, we carry out a linear stability analysis on the onset of thermal convection of a fluid with strongly temperature-dependent viscosity in a spherical shell geometry, in order to classify the convective flow patterns in terms of the changes in the vertical flow structures and, in particular, to find the condition for the dominance of the ST convection. We will begin by revisiting the analysis by [19] where the transition into the ST regimes has been identified in a theoretical manner for a convection in a planar layer. By carefully studying the vertical structures of incipient flows, we will first develop an empirical but quantitative criterion for the dominance of the ST convection, which is valid regardless of the model geometries. We will then apply the criterion to the convection in a spherical shell geometry in order to theoretically locate the transition into the ST regime. By further comparing the conditions for the changes in vertical and horizontal flow structures, we aim at deepening the insights into convective planforms in spherical shells in the presence of strongly temperature-dependent viscosity.

\section{Model descriptions}

In Fig. 1, we schematically show the conceptual models employed here. The onset of thermal convection is considered for a basally heated Boussinesq fluid of infinite Prandtl number with strongly temperature-dependent viscosity either in a planar layer or in a spherical shell whose temperatures are kept to be $T_{s}$ and $T_{b}$ at the top (inner) and bottom (outer) surfaces, respectively. The aspect ratio of the spherical shell is characterized by $\gamma \equiv r_{i} / r_{o}$, where $r_{i}$ and $r_{o}$ are the inner and outer radii of the shell, respectively. In order to focus on the 
(a) planar layer

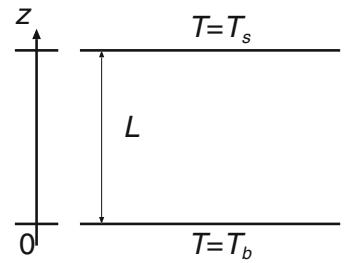

(b) spherical shell

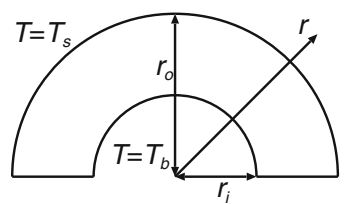

Fig. 1 Schematic illustration of the numerical model in this study

effects of temperature-dependent viscosity, the spatial variations in other physical quantities (such as thermal expansivity and thermal conductivity) are ignored.

In this study, we solved for the temporal evolution of an infinitesimal perturbation superimposed to a reference state described by a stationary (motionless) state with steady one-dimensional heat conduction in the vertical (radial) direction. In the following, the quantities with overbars and primes denote those of reference state and perturbation, respectively. The general forms of the dimensionless equations for the reference state are written as

$$
0=\nabla^{2} \bar{T}, \quad 0=\overline{\boldsymbol{v}}, \quad \bar{\eta}=\exp \left[-E\left(\bar{T}-\frac{1}{2}\right)\right],
$$

while those for infinitesimal perturbations are given by

$$
\begin{aligned}
0 & =\nabla \cdot \boldsymbol{v}^{\prime}, \\
0 & =-\nabla p^{\prime}+\nabla \cdot\left[\bar{\eta}\left(\nabla \otimes \boldsymbol{v}^{\prime}+\boldsymbol{v}^{\prime} \otimes \nabla\right)\right]+R a T^{\prime} \boldsymbol{e}_{g}, \\
\frac{\partial T^{\prime}}{\partial t} & =-\boldsymbol{v}^{\prime} \cdot \nabla \bar{T}+\nabla^{2} T^{\prime} .
\end{aligned}
$$

Here, $T$ is temperature, $\boldsymbol{v}$ is velocity vector, $\eta$ is viscosity, $p$ is pressure, and $\boldsymbol{e}_{g}$ is the unit vector in vertical (radial) direction positive upward (outward). In deriving these equations, the second-order terms of infinitesimal (primed) quantities are ignored, and the non-dimensionalization is carried out with the length scale of $L$ (三 $r_{o}-r_{i}$ for spherical shell) and temperature scale of $T_{b}-T_{s}$. Note also that the value of viscosity $\eta$ is scaled by $\eta_{\text {ref }}$, the value at non-dimensional temperature $T=\frac{1}{2}$. In these equations, the rheological properties of the system are expressed by two non-dimensional parameters. One is the Rayleigh number $R a$ of thermal convection defined by

$$
R a \equiv \frac{\rho_{\mathrm{ref}} \alpha\left(T_{b}-T_{S}\right) g L^{3}}{\eta_{\mathrm{ref}} \kappa},
$$

where $\rho_{\text {ref }}, g, \alpha$, and $\kappa$ are the reference values of density, gravity, thermal expansivity, and thermal diffusivity,

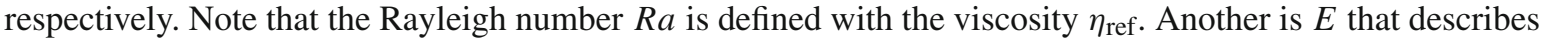
the magnitude of temperature dependence in viscosity. At the top and bottom boundaries, we kept the perturbations in temperature, $T^{\prime}$, and the normal velocity, $\boldsymbol{v}^{\prime} \cdot \boldsymbol{e}_{g}$, to be zero. For the shear velocity at the boundaries, on the other hand, we assumed either no-slip or free-slip conditions.

\subsection{Explicit forms of basic equations}

According to a general course of linear analysis [1, for example], the evolutionary equations for perturbations are solved by separation of variables into temporal, vertical (radial), and horizontal directions. In order to simplify the present analysis, we assume that the infinitesimal velocity vector, $\boldsymbol{v}^{\prime}$, consists only of poloidal field as:

$$
\boldsymbol{v}^{\prime}=\nabla \times \nabla \times\left(W^{\prime} \boldsymbol{e}_{g}\right),
$$

where $W^{\prime}$ is a potential for poloidal field. Note that the velocity field is free from the toroidal components, since there is no body force acting in the horizontal directions. 
Suppose, for a planar layer, the temporal and spatial dependence of perturbations is

$$
\left[\begin{array}{c}
T^{\prime}(x, y, z, t) \\
W^{\prime}(x, y, z, t)
\end{array}\right]=\left[\begin{array}{c}
\Theta(z) \\
W(z)
\end{array}\right] \exp \left[i\left(k_{x} x+k_{y} y\right)+a t\right] .
$$

Here, $a$ is the growth rate and $k_{x}$ and $k_{y}$ are the wavenumbers of perturbation in the $x$ and $y$ directions, respectively. By substituting the above equation into (2), (3), and (4), and eliminating pressure $p^{\prime}$, we obtain the equations for the vertical profiles of the perturbations in temperature $\Theta$ and poloidal potential of velocity $W$ as

$$
\begin{aligned}
a \Theta & =-\frac{\mathrm{d} \bar{T}}{\mathrm{~d} z} K^{2} W+\left(-K^{2}+\frac{\mathrm{d}^{2}}{\mathrm{~d} z^{2}}\right) \Theta \\
R a \Theta & =\bar{\eta}\left(-K^{2}+\frac{\mathrm{d}^{2}}{\mathrm{~d} z^{2}}\right)^{2} W+2 \frac{\mathrm{d} \bar{\eta}}{\mathrm{d} z}\left(-K^{2}+\frac{\mathrm{d}^{2}}{\mathrm{~d} z^{2}}\right) \frac{\mathrm{d} W}{\mathrm{~d} z}+\frac{\mathrm{d}^{2} \bar{\eta}}{\mathrm{d} z^{2}}\left(K^{2}+\frac{\mathrm{d}^{2}}{\mathrm{~d} z^{2}}\right) W
\end{aligned}
$$

where

$$
K \equiv \sqrt{k_{x}^{2}+k_{y}^{2}}
$$

is the horizontal wavenumber of perturbations, and the vertical profile of vertical velocity, $V$, with wavenumber $K$ is given by $V=K^{2} W$. The boundary conditions for $\Theta$ and $W$ are

$$
\begin{array}{ll}
\Theta=W=0 & \text { at } z=0,1 \\
\frac{\mathrm{d} W}{\mathrm{~d} z}=0 \quad \text { or } \frac{\mathrm{d}^{2} W}{\mathrm{~d} z^{2}}=0 & \text { at } z=0,1
\end{array}
$$

where $z=1$ and $z=0$ denote the top and bottom boundaries in non-dimensional units, respectively.

Similarly, for a spherical shell, we will obtain the equations for the radial profiles of the perturbations in temperature $\Theta$ and poloidal potential of velocity, $W$. By expanding the perturbations into spherical harmonics in the horizontal directions, we obtain the equations for $\Theta$ and $W$ of the spherical harmonics degree, $\ell$, as,

$$
\begin{aligned}
a \Theta= & -L \frac{1}{r^{2}} \frac{\mathrm{d} T}{\mathrm{~d} r} W+\frac{\mathrm{d}^{2} \Theta}{\mathrm{d} r^{2}}+\frac{2}{r} \frac{\mathrm{d} \Theta}{\mathrm{d} r}-L \frac{1}{r^{2}} \Theta \\
R a \Theta= & \bar{\eta}\left[\frac{\mathrm{d}^{4} W}{\mathrm{~d} r^{4}}-\frac{2}{r^{2}} L \frac{\mathrm{d}^{2} W}{\mathrm{~d} r^{2}}+\frac{4}{r^{3}} L \frac{\mathrm{d} W}{\mathrm{~d} r}+\frac{L(L-6)}{r^{4}} W\right] \\
& +2 \frac{\mathrm{d} \bar{\eta}}{\mathrm{d} r}\left[\frac{\mathrm{d}^{3} W}{\mathrm{~d} r^{3}}-\frac{1}{r} \frac{\mathrm{d}^{2} W}{\mathrm{~d} r^{2}}-(L-1) \frac{1}{r^{2}} \frac{\mathrm{d} W}{\mathrm{~d} r}+2 L \frac{1}{r^{3}} W\right] \\
& +\frac{\mathrm{d}^{2} \bar{\eta}}{\mathrm{d} r^{2}}\left(\frac{\mathrm{d}^{2} W}{\mathrm{~d} r^{2}}-\frac{2}{r} \frac{d W}{\mathrm{~d} r}+L \frac{1}{r^{2}} W\right)
\end{aligned}
$$

where $L \equiv \ell(\ell+1)$, and the radial profile of radial velocity $V$ with spherical harmonic degree $\ell$ is given by $V=\frac{L}{r^{2}} W$. Note also that there is no dependence on the order of the spherical harmonics in (13) or (14). The boundary conditions for $\Theta$ and $W$ are

$$
\begin{aligned}
& \Theta=W=0 \quad \text { at } r=r_{0}, r_{1} \\
& \frac{\mathrm{d} W}{\mathrm{~d} r}=0 \quad \text { or } \quad \frac{\mathrm{d}}{\mathrm{d} r}\left(\frac{1}{r^{2}} \frac{\mathrm{d} W}{\mathrm{~d} r}\right)=0 \text { at } r=r_{0}, r_{1}
\end{aligned}
$$

where $r=r_{0}$ and $r=r_{1}$ denote the inner and outer surfaces of spherical shell in non-dimensional units, respectively. 


\subsection{Numerical techniques}

In this article, we study the variation in the nature of the infinitesimal perturbations that become unstable for the smallest Rayleigh number by a following strategy. First, for given conditions (model geometry, boundary conditions, and temperature dependence of viscosity), we seek for a critical Rayleigh number, $R a_{c}$, of perturbations with wavenumber $K$ or spherical harmonic degree, $\ell$. In this procedure, we iteratively solved the eigenequations describing a critical state (i.e., growth rate $a=0)$ for an eigenvalue $\left(R a_{c}\right)$ together with eigenfunctions (vertical profiles of perturbations of temperature $\Theta$ and vertical velocity $V$ ). The details of the numerical technique can be found in Appendix. Among the series of eigenvalues and eigenfunctions in hand, we then search the one that yields the absolute minimum of the critical Rayleigh number, $R a_{c 0}$, and the wavenumber, $K_{c 0}$, or the spherical harmonic degree, $\ell_{c 0}$, corresponding to $R a_{c 0}$.

The differential equations for $\Theta$ and $W$ (Eqs. 8 and 9 for a planar layer, 13 and 14 for a spherical shell) are discretized with a sixth-order accuracy in space using equally spaced 257 grid points. As will be demonstrated in later sections, the reliability of the present numerical technique is verified by the comparison with the earlier results of $[12,19]$.

\section{Results}

\subsection{Control experiments: onset of convection in a planar layer}

We first carried out control experiments similar to the work by [19]: the onset of convection in a planar layer. In the following, we denote no-slip boundaries by R (rigid) and stress-free boundaries by F (free-slip) for convenience. We then use a fractional notation with the top of the fraction being the condition at the top boundary and the bottom of the fraction being the condition at the bottom boundary. Thus, F/R means that the top surface is free-slip and the bottom surface is rigid. We consider four cases: $F / F, R / R, F / R$, and R/F.

In Fig. 2, we show the plots of (a) the absolute minimum value of critical Rayleigh number, $R a_{c 0}$, and (b) the wavenumber, $K_{c 0}$, corresponding to $R a_{c 0}$, against $E$ for various boundary conditions indicated in the figure. From these experiments, we confirmed the earlier finding [19] that three regimes can be distinguished: (i) the regime with weakly temperature-dependent viscosity $(E \lesssim 1.5)$ where $R a_{c 0}$ and $K_{c 0}$ are nearly constant, (ii) that with moderate dependence $(1.5 \lesssim E \lesssim 8)$ where $R a_{c 0}$ increases and $K_{c 0}$ is nearly constant or decreases moderately depending on the boundary conditions, and (iii) that with strong dependence $(E \gtrsim 8)$ where $R a_{c 0}$ reaches a peak and then decreases, while $K_{c 0}$ rises rapidly. In particular, for the cases with free-slip top boundaries ( $\mathrm{F} / \mathrm{F}$ and $\mathrm{F} / \mathrm{R})$, the plots of $R a_{c 0}$ have maxima at around $E=8$, which has been commonly regarded as the transition into the "Stagnant-Lid" (ST) regime. Indeed, as can be seen from the figure, the

(a) $R a_{c 0}$ against $E$

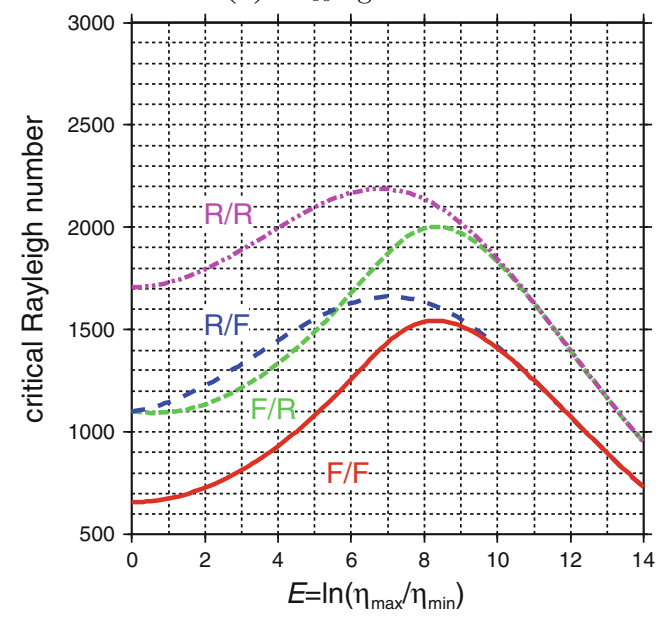

(b) $K_{c 0}$ against $E$

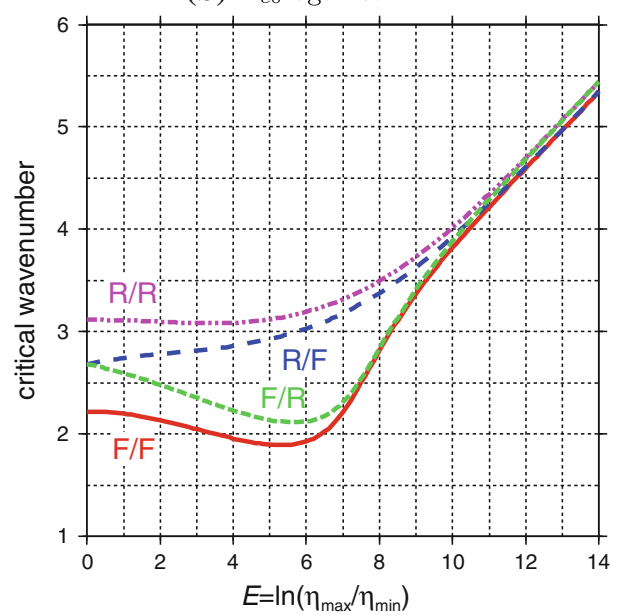

Fig. 2 The plots of a the absolute minimum value of critical Rayleigh number, $R a_{c 0}$, and $\mathbf{b}$ the wavenumber, $K_{c 0}$, corresponding to $R a_{c 0}$, against $E$ for various boundary conditions indicated in the figure (e.g., "R/F" for a rigid top and a free-slip bottom boundaries). Note that the Rayleigh number is defined with the viscosity for non-dimensional temperature $T=0.5$ 
(a) $\mathbf{F} / \mathbf{F}$

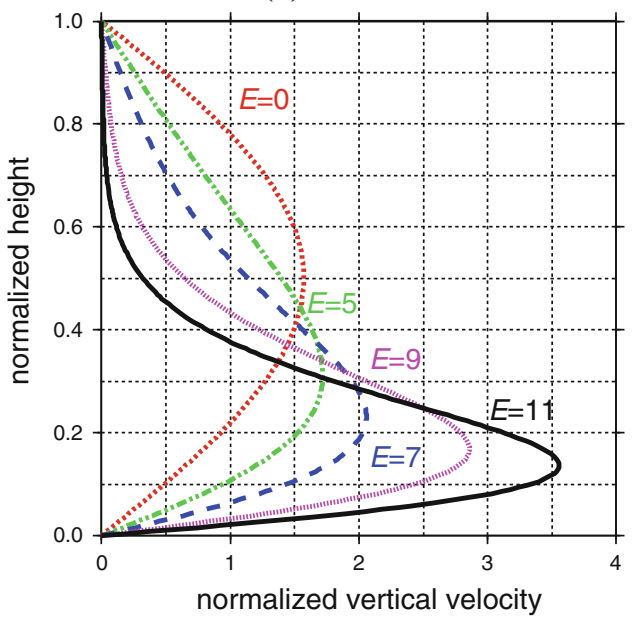

(b) $\mathbf{R} / \mathbf{F}$

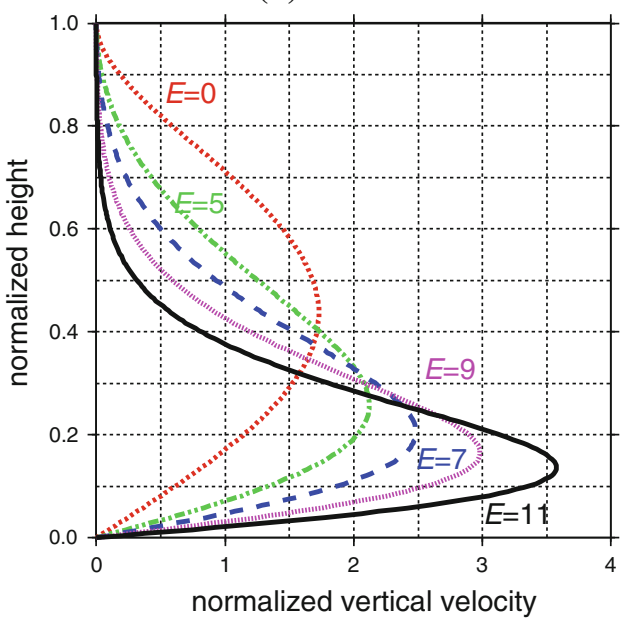

Fig. 3 Plots of vertical profiles of eigenfunctions for vertical velocity $V$ for $\mathbf{a} F / F$ and $\mathbf{b} R / F$ boundaries with various values of $E$ indicated in the figure. In each plot, the values of $V$ are normalized in order to set their volumetric average to unity

values of $R a_{c 0}$ are the largest at $E=8.3$ for both cases. Figure 2 also shows that for sufficiently large $E$, the plots for the case with free-slip top boundaries (F/F and F/R) become close to those for the corresponding case with rigid top boundaries (R/F and R/R). This is consistent with the nature of ST mode of convection, where the motion of the highly viscous fluid is negligibly small along the top cold surface boundary.

The transition into ST regime with increasing $E$ can also be observed from Fig. 3, where we compared the vertical profiles of eigenfunctions for vertical velocity $V$ for the critical states with various values of $E$ obtained with (a) F/F and (b) R/F boundary conditions. The plots of $V$ in the figure show that, as $E$ becomes larger, the values of $V$ become smaller and the positions of their local maxima become lower. In particular, for sufficiently large $E$, the profiles of eigenfunctions for vertical velocity, $V$, become almost identical both for the $\mathrm{F} / \mathrm{F}$ and $\mathrm{R} / \mathrm{F}$ cases (see, for example, the profiles with $E=11$ ), implying that the cold fluid behaves as a stagnant lid regardless of the boundary conditions along the top surface.

The fact that the transition into ST regime occurs at around $E \simeq 8$ has been analytically estimated by the earlier work by [19], based on an assumption that convection begins in a sublayer spanning $0 \leq z \leq \hat{z}(<1)$. They consider the "local" Rayleigh number of the sublayer $\hat{R a}$ given by,

$$
\hat{R a} \equiv \frac{\rho_{\mathrm{ref}} \alpha\left[\left(T_{b}-T_{S}\right) \hat{z}\right] g(L \hat{z})^{3}}{\hat{\eta}_{\mathrm{ref}} \kappa}=R a \frac{\eta_{\mathrm{ref}}}{\hat{\eta}_{\mathrm{ref}}} \hat{z}^{4},
$$

where $\left(T_{b}-T_{s}\right) \hat{z}$ is the temperature drop in the sublayer. By further taking the reference viscosity $\hat{\eta}_{\text {ref }}$ in the sublayer to be that at its mid-depth $\left(T=1-\frac{\hat{z}}{2}\right.$ at $\left.z=\frac{\hat{z}}{2}\right)$, the ratio of $\hat{R} a$ to $R a$ can be written as

$$
\frac{\hat{R a}}{R a}=\hat{z}^{4} \exp \left[E\left(\frac{1}{2}-\frac{\hat{z}}{2}\right)\right] .
$$

Differentiating the above equation with respect to $\hat{z}$ gives

$$
\frac{\partial}{\partial \hat{z}}\left(\frac{\hat{R a}}{R a}\right)=\left(\frac{\hat{R a}}{R a}\right)\left(\frac{4}{\hat{z}}-\frac{E}{2}\right),
$$

implying that for $E \geq 8$, there exists a sublayer with $\hat{z}=8 / E \leq 1$, which yields a maximum of $\hat{R a} / R a$ larger than 1 . This allows the onset of convection only in a sublayer that does not extend to the upper boundary. This estimate of threshold $E$ agrees well with our numerical finding that the $R a_{c 0}$ has a maximum at around $E=8.3$.

As has already been demonstrated in the earlier work [19], the validity of the above analysis can be also confirmed from the variations of the absolute minimum value of critical "local" Rayleigh number $\hat{R a_{c 0}}$ defined 


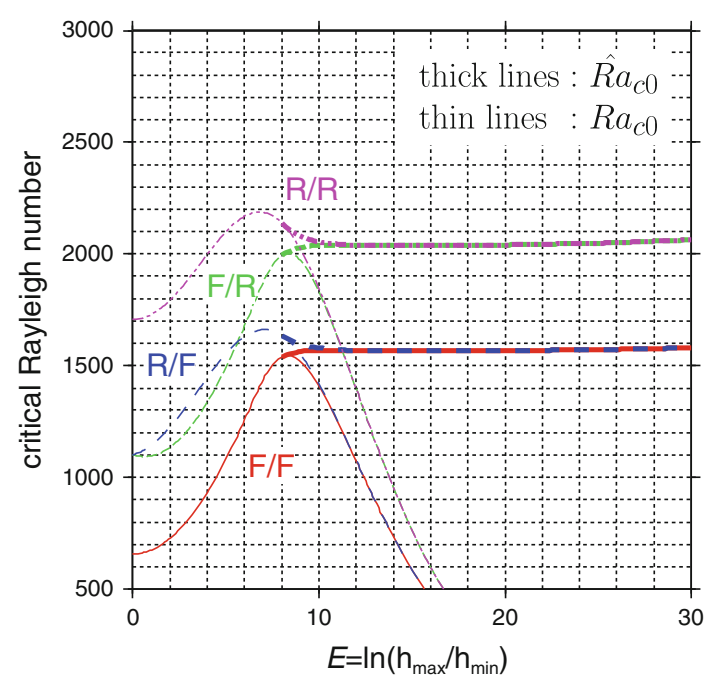

Fig. 4 The plots against $E$ of the absolute minimum value of critical "local" Rayleigh number $\hat{R} a_{c 0}$ for the stagnant-lid (ST) regime ( $E \geq 8$; thick lines). The meanings of the labels for the boundary conditions are the same as in Fig. 2. For comparison, we also plotted against $E$ the values of $R a_{c 0}$ by thin lines

by

$$
\hat{R} a_{c 0} \equiv R a_{c 0} \hat{z}_{c 0}^{4} \exp \left[E\left(\frac{1}{2}-\frac{\hat{z}_{c 0}}{2}\right)\right]
$$

where $\hat{z}_{c 0}=8 / E$. Namely, $\hat{R a} a_{c 0}$ is the "local" Rayleigh number, corresponding to $R a_{c 0}$, of the basal sublayer whose $\hat{R a}$ is maximum. We show in Fig. 4 the plots against $E$ the values of $R a_{c 0}$ over a broad range of $E \leq 30$ (or, the viscosity contrast of $\exp (30) \simeq 10^{13}$ of the entire layer). As can be seen from the plots, the values of $\hat{R} a_{c 0}$ are always larger than those of $R a_{c 0}$ for the ST convection (see also 18). In addition, according to the assumption that the onset of convection occurs only in a basal sublayer, the values of $\hat{R a} c_{c 0}$ are expected to be constant in the stagnant-lid regime. The plots in the figure clearly show that the above conjecture is valid for the range of $E$ studied here. The asymptotic values of $\hat{R} a_{c 0}$ are around 2,038 and 1,568 when the lower boundary is rigid and free-slip, respectively.

In order to quantitatively study the changes in the vertical flow structures toward the dominance of the ST convection, we consider a quantity $\Delta_{h}$ defined by

$$
\begin{aligned}
\frac{\partial v_{x}}{\partial x}+\frac{\partial v_{y}}{\partial y}=-\frac{\partial v_{z}}{\partial z} & =-\frac{\mathrm{d} V}{\mathrm{~d} z} \exp \left[i\left(k_{x} x+k_{y} y\right)+a t\right] \\
& \equiv \Delta_{h}(z) \exp \left[i\left(k_{x} x+k_{y} y\right)+a t\right] .
\end{aligned}
$$

By definition, the quantity $\Delta_{h}(z)$ is a measure of the divergence of horizontal velocity $\left(v_{x}\right.$ and $\left.v_{y}\right)$ in the horizontal plane at a non-dimensional height $z$. Note that the value of $\Delta_{h}(z)$ is also a measure of the extent of conversion between horizontal and vertical flows at a height $z$. This indicates that the source regions of ascending and descending flows can be estimated from its vertical profiles. In Fig. 5, we show the vertical profiles of $\Delta_{h}(z)$ for various values of $E$ obtained for the case with $\mathrm{F} / \mathrm{F}$ boundary condition. The vertical profile of $\Delta_{h}$ for an isoviscous case $(E=0)$ is symmetric with respect to the mid-depth of the layer $(z=0.5)$, with two maxima at top and bottom boundaries $(z=0$ and $z=1)$. In addition, the minimum at $z=0.5$ comes from the fact that the eigenfunction of vertical velocity $V$ has a maximum at the depth.

As can be seen from the changes in the vertical profiles of $\Delta_{h}$ with increasing $E$ in Fig. 5, the transition in the convective regimes can be identified by the difference in the positions and magnitudes of the local maxima of $\Delta_{h}$ occurring in the upper and colder part of the layer (i.e., the maxima other than that at $z=0$ ). The vertical profiles of $\Delta_{h}(z)$ for $E \lesssim 3$ have maxima at $z=1$. This is consistent with the feature of so-called whole-layer (WH) mode of convection $[3,15]$ occurring with a weakly temperature-dependent viscosity, where the coldest fluid along the top surface is involved in an overall convective motion. For $E=5$, in contrast, the profile of $\Delta_{h}$ has a local maximum in the mid-depth at around $z=0.6$. This indicates that the motion of the highly viscous 


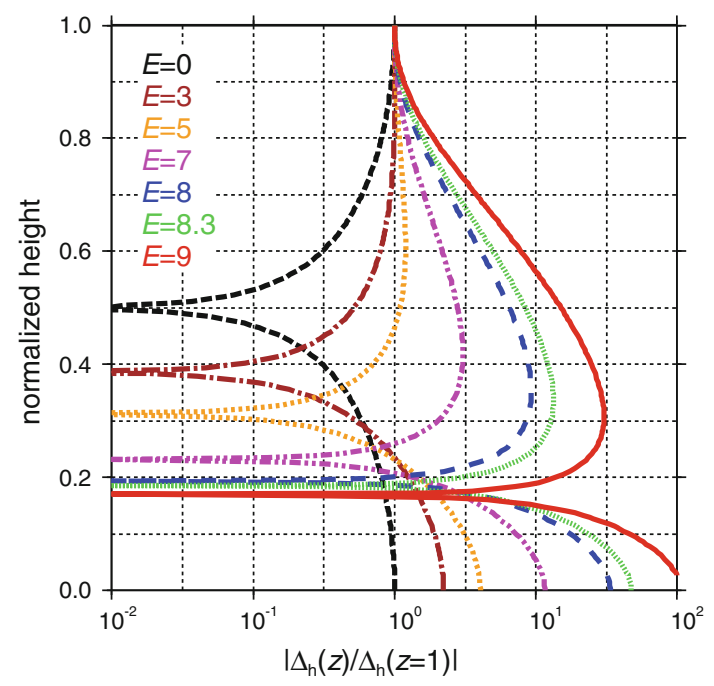

Fig. 5 Plots of the vertical profiles of $\Delta_{h}$, a measure of conversion between horizontal/vertical flows, for various values of $E$ indicated in the figure. Shown are the results of the cases with F/F boundaries. Note that plotted are the absolute values of the ratios of $\Delta_{h}$ to the values at the top surface $(z=1)$

fluid in the uppermost part of the layer is slightly decoupled from that of less viscous fluid in the interior. As $E$ increases further, the local maximum occurs at a deeper part of the layer, and the value of local maximum $\Delta_{h \max }$ becomes larger. When the ST convection becomes dominant $(E=8.3$; see also Fig. 2$)$, the value of $\Delta_{h \max }$ becomes more than 10 times larger than that of $\Delta_{h}(z=1)$. This indicates that the fluid motion becomes negligible in the uppermost part of the layer, and, in other words, a stagnant lid of cold and highly viscous fluid develops along the top surface boundary.

In summary, the present numerical method has successfully reproduced the change in the style of convection depending on the changes in temperature-dependent viscosity. The transition can be identified by the quantity $\Delta_{h}$, which is a measure of the extent of conversion between horizontal and vertical flow at a particular height. In addition, we found that, when the stagnant-lid (ST) convection becomes dominant, the vertical profiles of $\Delta_{h}(z)$ have local maxima in the mid-depth whose magnitude is more than 10 times larger than that at the top surface $(z=1)$. From this finding, we will identify in the next section, the transition in convective patterns in the spherical shell geometry based on the vertical profiles of $\Delta_{h}$.

\subsection{Onset of convection in spherical shell geometries}

In this section, we will study the onset of convection in a spherical shell of a fluid with temperature-dependent viscosity.

First we verified the validity of the present technique, by comparing our numerical results with those of an earlier study by [12]. Here we calculated the values of the critical Rayleigh number, $R a_{c}$, in a spherical shell with $\gamma=0.55$, by varying the spherical harmonic degrees, $\ell$, up to 6 and the viscosity contrast due to the temperature dependence $r_{\eta} \equiv \exp (E)$ up to $10^{3}$. In Table 1, we summarize the comparison of the present results with those by [12]. As can be seen in Table 1, the values of $R a_{c}$ obtained from two different studies show an excellent agreement for the ranges of $\ell$ and $r_{\eta}$ considered here. From this comparison, we conclude that our technique successfully captures the critical state of convection in the spherical shell geometry.

In the rest of this section, we will study the condition for the stagnant-lid (ST) convection for the spherical shell geometry by varying the magnitude of the temperature-dependent viscosity, $E$. In particular, we will focus on how the transition is affected by the model geometry, including the ratio of the inner and outer radii of the spherical shell, $\gamma$.

\subsubsection{Empirical estimates for the condition for the ST-mode for spherical shell geometry}

To clearly show the differences from the results of the control experiments, we will start with a comparison of the cases with thin (aspect ratio $\gamma=0.95)$ and thick $(\gamma=0.55)$ spherical shells. Note that a model with 
Table 1 The values of the critical Rayleigh number of the fluid with a temperature-dependent viscosity in a spherical shell of $\gamma=0.55$ whose inner and outer surfaces are free-slip boundaries

\begin{tabular}{|c|c|c|c|c|c|c|c|}
\hline \multirow{2}{*}{\multicolumn{2}{|c|}{$\overline{r_{\eta}}=\exp (E)$}} & \multicolumn{6}{|c|}{ Spherical harmonic degree $\ell$} \\
\hline & & 1 & 2 & 3 & 4 & 5 & 6 \\
\hline $10^{0}$ & $\begin{array}{l}\text { This study } \\
{[12]}\end{array}$ & $\begin{array}{l}1,864.34 \\
1,864.34\end{array}$ & $\begin{array}{l}888.84 \\
888.84\end{array}$ & $\begin{array}{l}711.95 \\
711.95\end{array}$ & $\begin{array}{l}729.39 \\
729.38\end{array}$ & $\begin{array}{l}851.31 \\
851.31\end{array}$ & $\begin{array}{l}1,063.95 \\
1,063.95\end{array}$ \\
\hline $10^{1}$ & $\begin{array}{l}\text { This study } \\
{[12]}\end{array}$ & $\begin{array}{l}2,136.64 \\
2,136.65\end{array}$ & $\begin{array}{l}1,085.05 \\
1,085.05\end{array}$ & $\begin{array}{l}913.73 \\
913.73\end{array}$ & $\begin{array}{l}963.06 \\
963.07\end{array}$ & $\begin{array}{l}1,135.81 \\
1,135.81\end{array}$ & $\begin{array}{l}1,418.09 \\
1,418.09\end{array}$ \\
\hline $10^{2}$ & $\begin{array}{l}\text { This study } \\
{[12]}\end{array}$ & $\begin{array}{l}2,313.50 \\
2,313.49\end{array}$ & $\begin{array}{l}1,456.04 \\
1,456.04\end{array}$ & $\begin{array}{l}1,387.90 \\
1,387.91\end{array}$ & $\begin{array}{l}1,535.19 \\
1,535.20\end{array}$ & $\begin{array}{l}1,811.00 \\
1,811.01\end{array}$ & $\begin{array}{l}2,199.59 \\
2,199.60\end{array}$ \\
\hline $10^{3}$ & $\begin{array}{l}\text { This study } \\
{[12]}\end{array}$ & $\begin{array}{l}2,664.01 \\
2,663.98\end{array}$ & $\begin{array}{l}2,196.40 \\
2,196.39\end{array}$ & $\begin{array}{l}2,248.43 \\
2,248.43\end{array}$ & $\begin{array}{l}2,457.30 \\
2,457.29\end{array}$ & $\begin{array}{l}2,762.52 \\
2,762.52\end{array}$ & $\begin{array}{l}3,147.64 \\
3,147.63\end{array}$ \\
\hline
\end{tabular}

In the table, we compare the values obtained in this study and those obtained by [12], for various values of spherical harmonic degrees $\ell$ of the infinitesimal perturbations and the viscosity contrast due to the temperature dependence $r_{\eta} \equiv \exp (E)$

$\gamma=0.95$ is characterized by a volumetric average with a reference temperature of $T_{\mathrm{av}}=\frac{551}{1,141} \simeq 0.4829$ and inner and outer radii of $r_{0}=19$ and $r_{1}=20$, respectively. On the other hand, a shell with $\gamma=0.55$ has $r_{0}=11 / 9, r_{1}=20 / 9$, and $T_{\mathrm{av}}=\frac{77}{247} \simeq 0.3117$. In Fig. 6, we show the plots of (a) the absolute minimum value of critical Rayleigh number, $R a_{c 0}$, and (b) the degree of spherical harmonics, $\ell_{c 0}$, of infinitesimal perturbation corresponding to $R a_{c 0}$, against $E$ obtained both for the cases with $\gamma=0.95$ and $\gamma=0.55$ for various boundary conditions indicated in the figure.

A qualitative comparison between the results presented in Figs. 2 and 6 shows that the changes in the convective regimes for both $\gamma$ are similar with those obtained for a planar layer. Indeed, from the changes in $R a_{c 0}$ and $\ell_{c 0}$, three regimes can be distinguished depending on $E$ : (i) the regime with small $E$ where $R a_{c 0}$ and $\ell_{c 0}$ are nearly constant, (ii) that with moderate $E$ where $R a_{c 0}$ increases and $\ell_{c 0}$ is nearly constant or decreases moderately depending on the boundary conditions, and (iii) that with large $E$ where $R a_{c 0}$ reaches a peak and then decreases, while $\ell_{c 0}$ rises rapidly. In addition, for sufficiently large $E$, the plots for the case with free-slip top boundaries (F/F and F/R) become close to those for the corresponding case with rigid top boundaries $(\mathrm{R} / \mathrm{F}$ and $\mathrm{R} / \mathrm{R}$ ). This indicates that the ST mode of convection emerges in spherical shell geometries for $\gamma=0.95$ and $\gamma=0.55$ as well as in a planar layer.

However, a detailed comparison of the variations in $R a_{c 0}$ presented in Fig. 6a illuminates a quantitative difference in the changes in the convective regimes depending on the aspect ratio of the spherical shell $\gamma$. For the case with a thin spherical shell $(\gamma=0.95)$, the variations in $R a_{c 0}$ are very similar to those obtained in a planar layer throughout the range of $E$ studied here (see also Fig. 2). In particular, for the cases with free-slip top boundaries $(\mathrm{F} / \mathrm{F}$ and $\mathrm{F} / \mathrm{R})$, the plots of $R a_{c 0}$ have maxima at around $E \simeq 8$. For the case with a thick spherical shell $(\gamma=0.55)$, in contrast, the variations in $R a_{c 0}$ are significantly different from those for a planar layer. For a given $E$, the values of $R a_{c 0}$ are larger than those obtained for a planar layer regardless of the boundary conditions. In addition, the maxima in the plots of $R a_{c 0}$ against $E$ occur at larger values of $E$ than those for a planar layer and for a spherical shell with $\gamma=0.95$ (Note that, as will be discussed later in detail, the maxima in the plots of $R a_{c 0}$ do not necessarily imply the dominance of the ST convection).

We can also easily notice from Fig. $6 \mathrm{~b}$ that the aspect ratio of the spherical shell $\gamma$ affects the smoothness of the changes in convective regimes. The changes in $R a_{c 0}$ occur very smoothly and continuously with increasing $E$ for $\gamma=0.95$, while they occur in a quite discontinuous manner for $\gamma=0.55$. This comes from the differences in the thickness of the spherical shell and, in other words, the horizontal extent of the convecting layer compared to the vertical (radial) one. Indeed, the values of $\ell_{c 0}$ are large $\left(\ell_{c 0} \gtrsim 30\right)$ for $\gamma=0.95$, while they are small $\left(\ell_{c 0} \lesssim 10\right)$ for $\gamma=0.55$. Since the horizontal length scale of perturbation is roughly proportional to the reciprocal of spherical harmonic degree $\ell$, the effect of the change in $\ell$ is minor for sufficiently large $\ell$.

In order to precisely locate the dominance of the ST convection for spherical shell geometry, we consider the radial profile of $\Delta_{h}$, a measure of the extent of conversion between horizontal and vertical flows. Now in the spherical geometry, the quantity $\Delta_{h}$ is calculated from the eigenfunction for radial velocity $V$ (or that for its poloidal potential $W$ ) by

$$
\begin{aligned}
\frac{1}{r \sin \theta} \frac{\partial}{\partial \theta}\left(\sin \theta v_{\theta}\right)+\frac{1}{r \sin \theta} \frac{\partial v_{\phi}}{\partial \phi}=-\frac{1}{r^{2}} \frac{\partial}{\partial r}\left(r^{2} v_{r}\right) & =-\frac{1}{r^{2}} \frac{\mathrm{d}}{\mathrm{d} r}\left(r^{2} V\right) Y^{\ell m} \\
& \equiv \Delta_{h}(r) Y^{\ell m},
\end{aligned}
$$


(a) $R a_{c 0}$ against $E$

(b) $\ell_{c 0}$ against $E$
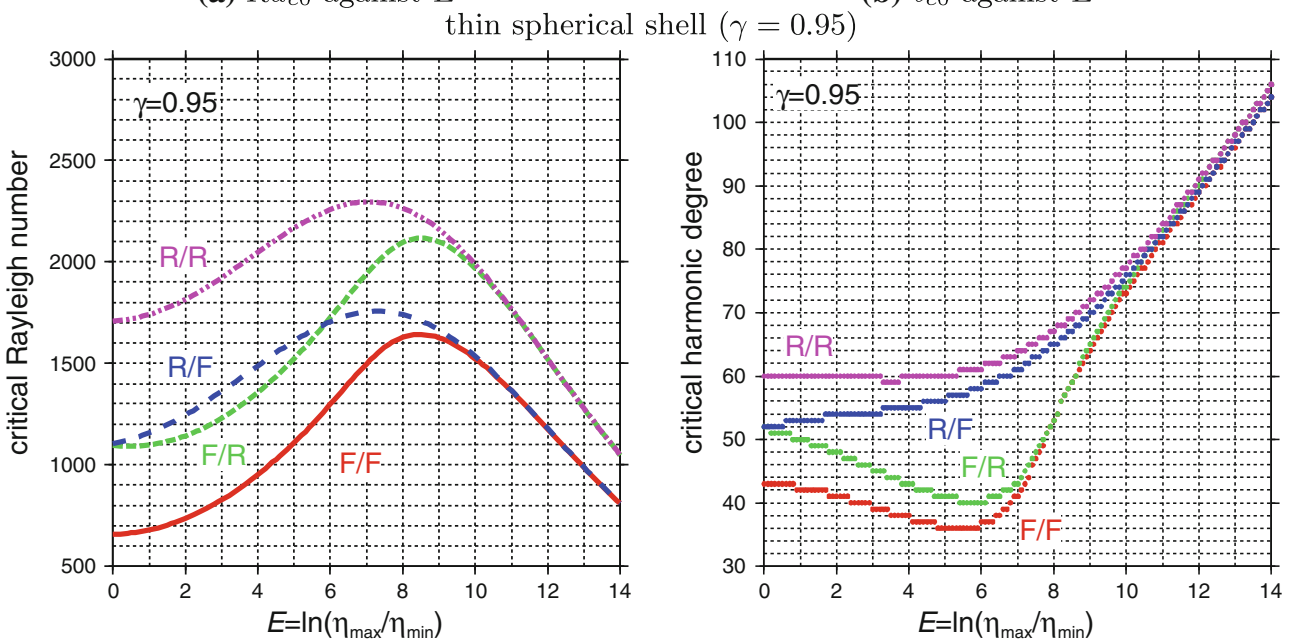

thick spherical shell $(\gamma=0.55)$
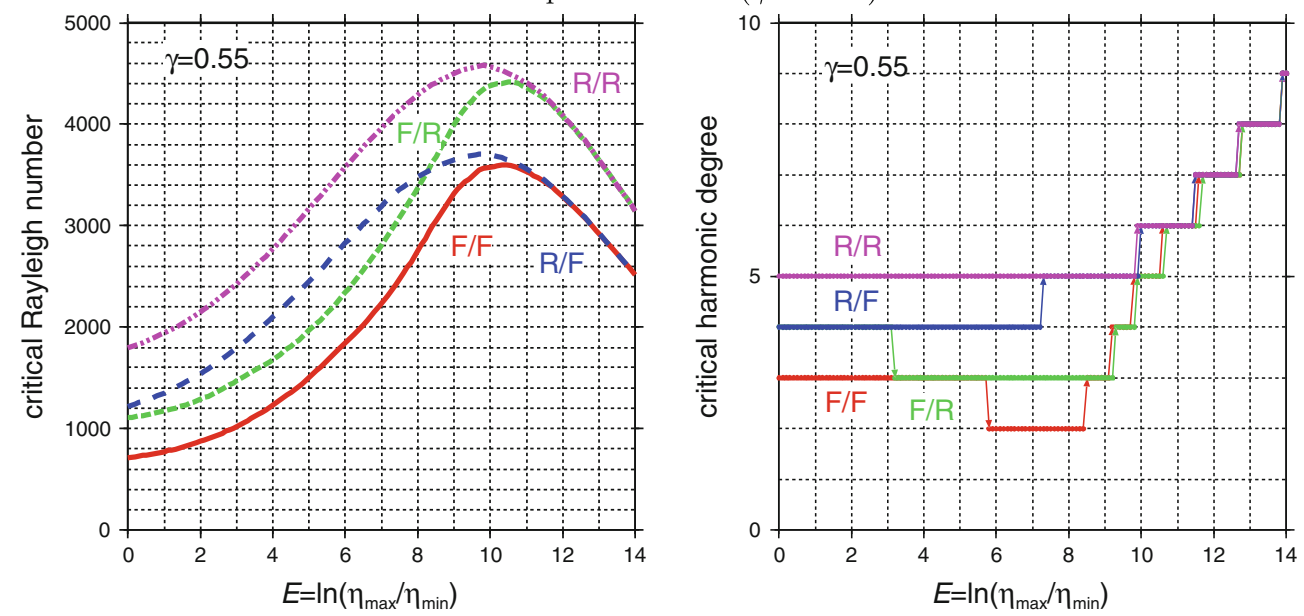

Fig. 6 The plots of a the absolute minimum value of critical Rayleigh number $R a_{c 0}$ and $\mathbf{b}$ the degree of spherical harmonics $\ell_{c 0}$ of infinitesimal perturbation corresponding to $R a_{c 0}$ against $E$ for various boundary conditions indicated in the figure (e.g., "R/F" for a rigid top and a free-slip bottom boundaries). Shown are the cases with thin $(\gamma=0.95 ;$ top $)$ and thick $(\gamma=0.55 ;$ bottom $)$ spherical shells. Note again that the Rayleigh number is defined with the viscosity for non-dimensional temperature $T=0.5$, which is of little significance to the dominance of the ST convection in a spherical shell geometry (see Sect. 3.2.2 for details)

where $Y^{\ell m}$ is the spherical harmonics with degree $\ell$ and order $m$. Note the difference in the definition of $\Delta_{h}$ in (22) from that for a planar layer (see also 21). We show in Fig. 7 the radial profiles of $\Delta_{h}(r)$ for various values of $E$ and the boundary condition of $\mathrm{F} / \mathrm{F}$ obtained for $\gamma=0.95$ and $\gamma=0.55$, while Fig. 8 are those of $V(r)$. Also shown in the figures are the values of $\ell_{c 0}$ of the perturbations for the corresponding cases.

The comparison of the radial profiles of $\Delta_{h}(r)$ in Fig. 7 clearly shows that the condition for the dominance of the ST-mode of convection is affected by the aspect ratio of the spherical shell $\gamma$. For $\gamma=0.95$, the transition occurs in a quite similar manner to the case with a planar layer (see also Fig. 5): at $E=8.3$ the local maximum of $\Delta_{h}(r)$ occurs at around $r-r_{0} \simeq 0.3$, and its value $\Delta_{h \max }$ becomes more than ten times larger than the value of $\Delta_{h}(r)$ at the top surface $\left(r=r_{1}\right)$. For $\gamma=0.55$, in contrast, the ratio of $\Delta_{h \max }$ to $\Delta_{h}\left(r=r_{1}\right)$ do not exceed the threshold value of $\sim 10$ unless $E$ becomes as large as around 9.2, implying that the dominance of the $\mathrm{ST}$ convection takes place at around $E=9.2$.

We also note that the transition is accompanied by the changes in $\ell_{c 0}$, which significantly affect the vertical profiles of $\Delta_{h}$ across the transition for small $\gamma$. This is mainly because the values of $\ell_{c 0}$ are smaller for $\gamma=0.55$ than for $\gamma=0.95$. As can be seen from Fig. 8, the change in $\ell_{c 0}$ causes a significant change in the radial profiles of $V$ when $\ell_{c 0}$ is small. 
(a) $\gamma=0.95$

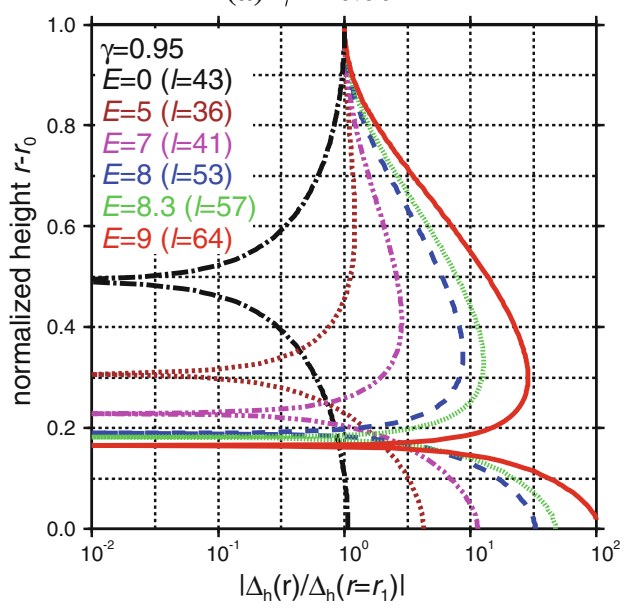

(b) $\gamma=0.55$

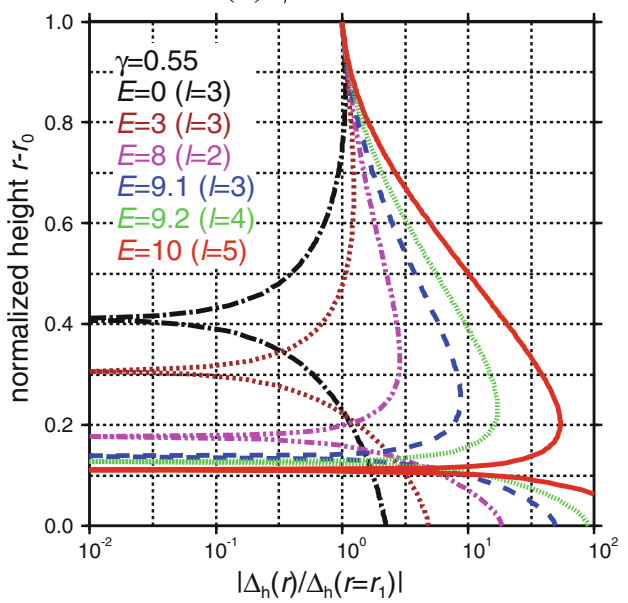

Fig. 7 Plots of the radial profiles of $\Delta_{h}$ for various values of $E$ indicated in the figure for $\mathbf{a}$ thin $(\gamma=0.95)$ and $\mathbf{b}$ thick $(\gamma=0.55)$ spherical shells. Also indicated in the figure are the values of $\ell_{c 0}$ of the perturbations. Shown are the results of the cases with $\mathrm{F} / \mathrm{F}$ boundaries. Note that plotted are the absolute values of the ratios of $\Delta_{h}$ to the values at the outer surface $\left(r=r_{1}\right)$ against the non-dimensional height from the inner surface $\left(r=r_{0}\right)$

(a) $\gamma=0.95$

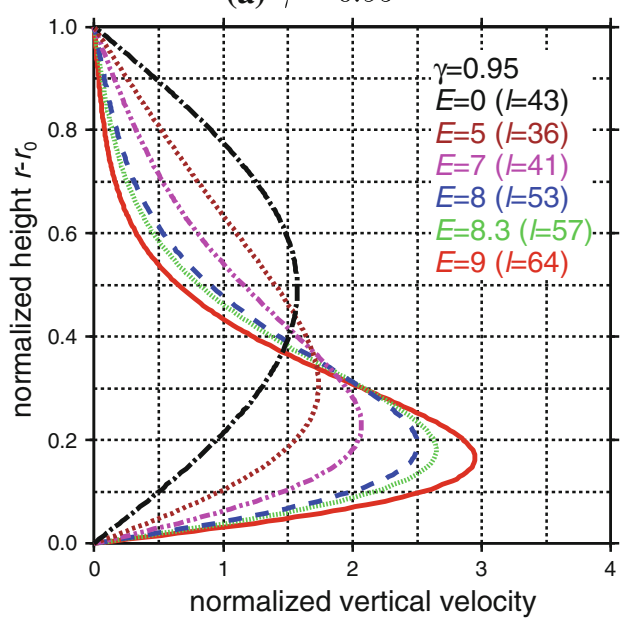

(b) $\gamma=0.55$

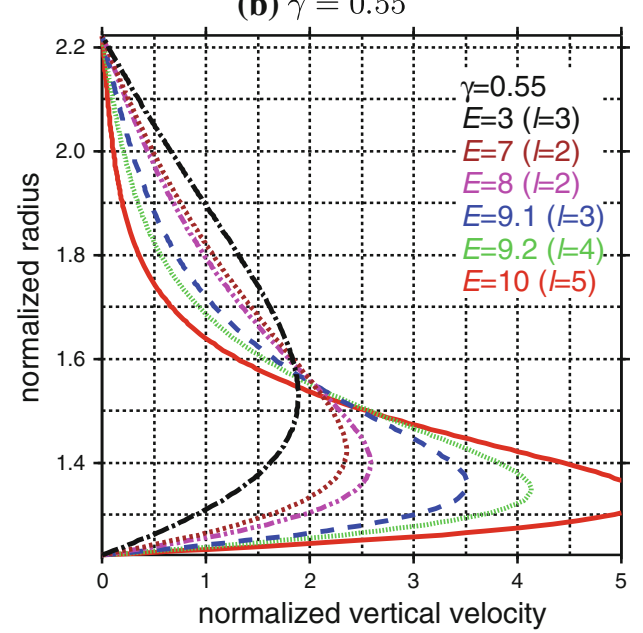

Fig. 8 Plots of vertical profiles of eigenfunctions for vertical velocity $V$ for spherical shells with $\mathbf{a} \gamma=0.95$ and $\mathbf{b} \gamma=0.55$ with various values of $E$ indicated in the figure. Also indicated in the figure are the values of $\ell_{c 0}$ of the perturbations. Shown are the results of the cases with $\mathrm{F} / \mathrm{F}$ boundaries plotted against the non-dimensional height from the inner surface $\left(r=r_{0}\right)$. In each plot, the values of $V$ are normalized in order to set their volumetric averages to unity

Table 2 The values of $E$ and $\ell_{c 0}$ at the dominance of the ST-mode of convection obtained for various values of the aspect ratio of the spherical shell

\begin{tabular}{lcccccccccccc}
\hline$\gamma$ & 0.95 & 0.9 & 0.85 & 0.8 & 0.75 & 0.7 & 0.65 & 0.6 & 0.55 & 0.5 & 0.45 & 0.4 \\
\hline$E$ & 8.3 & 8.3 & 8.3 & 8.4 & 8.4 & 8.5 & 8.8 & 8.9 & 9.2 & 9.3 & 9.8 & 10.5 \\
$\ell_{c 0}$ & 57 & 27 & 17 & 12 & 9 & 7 & 6 & 4 & 4 & 3 & 2 & 2 \\
\hline
\end{tabular}

We carried out similar calculations by varying the aspect ratio of the spherical shell $\gamma$ in the range of $0.4 \leq \gamma \leq 0.95$ in order to study how the conditions for the dominance of the ST convection are affected by the variation in $\gamma$. We show in Table 2 the values of $E$ and $\ell_{c 0}$ for the transition with various values of $\gamma$. Table 2 clearly shows that, for the range of $\gamma$ studied here, the dominance of the ST-mode of convection occurs at a larger $E$ for smaller $\gamma$. We also note that, for smaller $\gamma$, the values of $\ell_{c 0}$ for the perturbations become smaller at the dominance of the ST-mode. This is because the horizontal extent of the spherical shell becomes small compared to the vertical (radial) one for smaller $\gamma$. 


\subsubsection{Analytical estimates for the condition for the transition into ST-mode for spherical shell geometry}

In Sect. 3.2.1, we have numerically estimated the condition for the dominance of the ST-mode of convection in a spherical shell geometry, based on the changes in radial structures of the incipient flows. We found that the transition occurs in a quite different manner from that in a planar layer. In this section, we will analytically explore the condition for the transition into the ST regime in a spherical shell. In particular, we will demonstrate that a slight modification of the idea by [19], which has been used for a planar layer in Sect. 3.1, successfully reproduces the values of $E$ for the transition and their dependence on the aspect ratio of the spherical shell $\gamma$.

We consider a ST-mode of convection in a spherical shell whose inner and outer radii are $r_{0}$ and $r_{1}$ in non-dimensional units, respectively. Suppose that, following the idea by [19], the convecting instability grows only in a basal sublayer spanning $r_{0} \leq r \leq r_{2}$ (where $r_{0}<r_{2} \leq r_{1}$ ) and $T_{2} \leq \bar{T} \leq 1$ (where $T_{2}$ is the value of $\bar{T}$ at $r=r_{2}$ ). In addition, we consider two "modified" Rayleigh numbers $R a_{*}$ and $R a_{2 *}$; the former is for the entire layer and the latter is local in the lower sublayer. Note that one needs to estimate $R a_{*}$ and $R a_{2 *}$ based on a definition other than that employed so far, since the dominance of the ST convection can hardly be observed from the values of $R a$ used earlier (see also Fig. 6). In other words, by appropriately choosing $R a_{*}$ and $R a_{2 *}$, the regime for ST-mode of convection can be characterized by the existence of $r_{2}$, which satisfies $R a_{*}<R a_{2 *}$.

In Fig. 9, we schematically show the key assumption employed here for an appropriate choice of the Rayleigh number for a fluid with temperature-dependent viscosity in a spherical shell geometry. The "modified" Rayleigh number $R a_{*}$ for the entire layer is defined with the value of viscosity of temperature $T=T_{*}$ at $r=r_{*}$ given by

$$
\begin{aligned}
R a_{*} & \equiv \frac{\rho_{\mathrm{ref}} \alpha\left(T_{b}-T_{S}\right) g L^{3}}{\eta_{\mathrm{ref}} \exp \left[-E\left(T_{*}-\frac{1}{2}\right)\right] \kappa}=R a \exp \left[E\left(T_{*}-\frac{1}{2}\right)\right], \\
r_{*} & \equiv \sqrt{r_{0} r_{1}} \\
T_{*} & \equiv \frac{r_{0} r_{1}}{r_{1}-r_{0}}\left(\frac{1}{r_{*}}-\frac{1}{r_{1}}\right)=\frac{\sqrt{\gamma}-\gamma}{1-\gamma},
\end{aligned}
$$

respectively. As can be seen from Fig. 9a, the value of $r_{*}$ is chosen to be the radius in the spherical shell where the radial temperature gradient is equal to an average temperature gradient across the entire layer. Indeed, by using

$$
\bar{T}=\frac{r_{0} r_{1}}{r_{1}-r_{0}}\left(\frac{1}{r}-\frac{1}{r_{1}}\right), \quad \frac{\mathrm{d} \bar{T}}{\mathrm{~d} r}=-\frac{r_{0} r_{1}}{r_{1}-r_{0}} \frac{1}{r^{2}}
$$

(a) $r_{*}$ and $T_{*}$ (in entire layer)

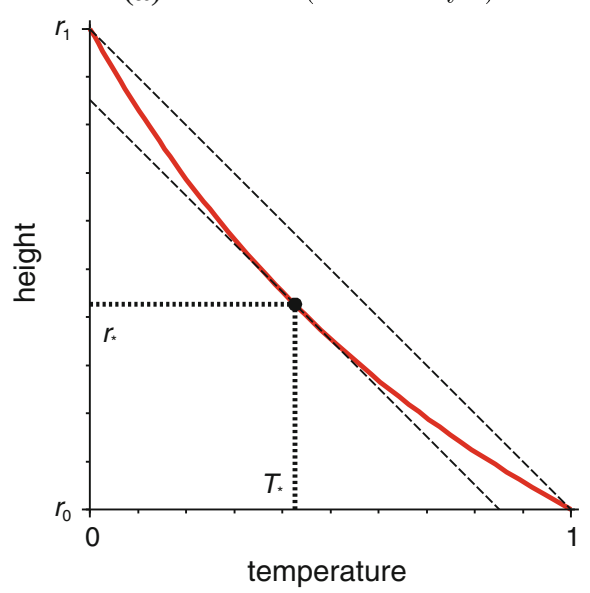

(b) $r_{2 *}$ and $T_{2 *}$ (in lower sublayer)

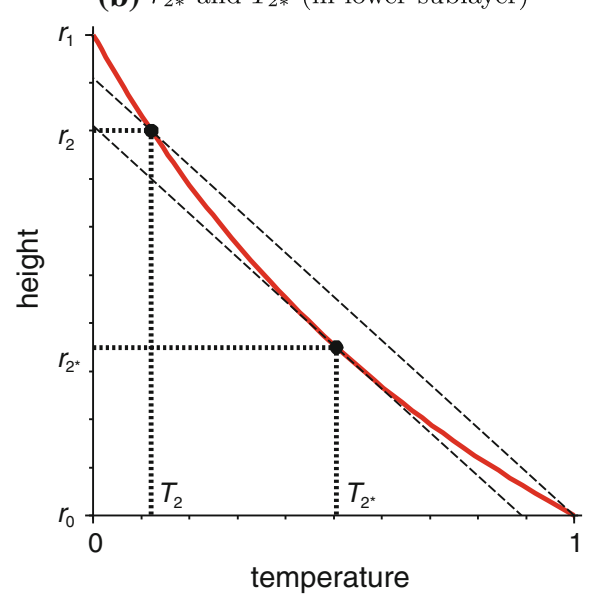

Fig. 9 a Schematic illustration of the meanings of "representative" radius $r_{*}$ and temperature $T_{*}$ used for a definition of Rayleigh number for a convection of a fluid with temperature-dependent viscosity in a spherical shell geometry. $\mathbf{b}$ The same as a but for the convection in a lower sublayer relevant to the ST-mode of convection 
(a) $r_{*}-r_{0}$ against $\gamma$

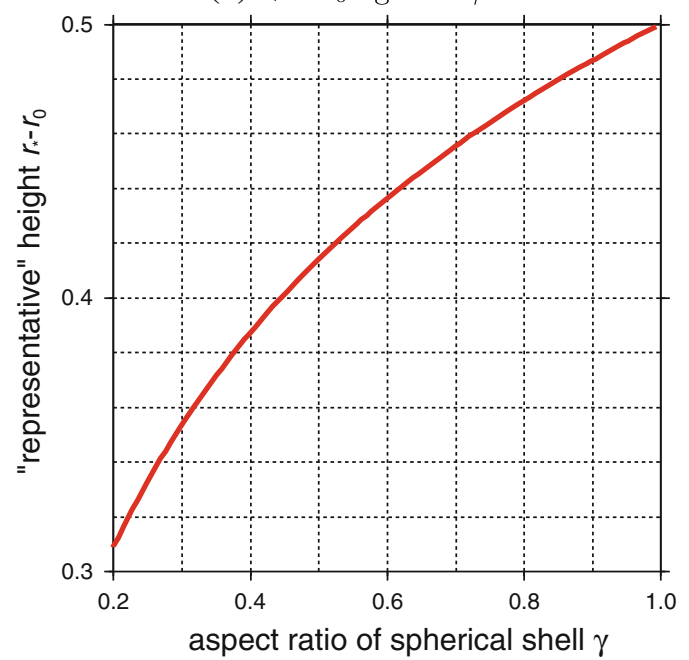

(b) $T_{*}$ against $\gamma$

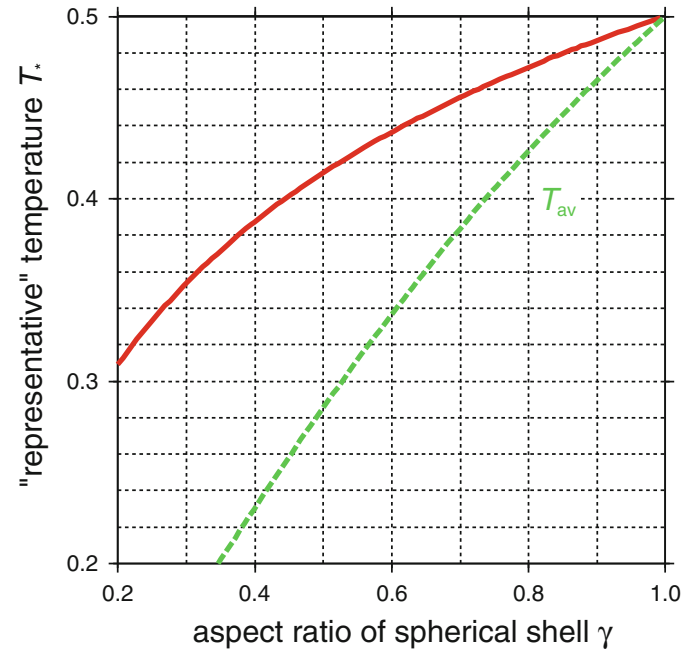

Fig. 10 The plots against the aspect ratio of the spherical shell $\gamma$ of the a position $r_{*}$ and $\mathbf{b}$ temperature $T_{*}$ which yield a "representative" viscosity. Also plotted in $\mathbf{b}$ is the values of volumetric average of temperature $T_{\mathrm{av}}$ of the reference state

we obtain $\frac{\mathrm{d} \bar{T}}{\mathrm{~d} r}=-1$ at $r=r_{*}$. We show in Fig. 10, the variations of $r_{*}$ and $T_{*}$ depending on the aspect ratio of the spherical shell $\gamma$. As can be seen from the figure, both of the values of $r_{*}$ and $T_{*}$ decrease as $\gamma$ decreases from 1. In particular, the value of $T_{*}$ significantly deviates from 0.5 with decreasing $\gamma$, implying a greater difference between $R a$ and $R a_{*}$ for smaller $\gamma$. Similarly, as indicated in Fig. 9b, let us define the local Rayleigh number $R a_{2 *}$ for the basal sublayer with the value of viscosity of $T=T_{2 *}$ at $r=r_{2 *}$ given by

$$
r_{2 *} \equiv \sqrt{r_{0} r_{2}} \text {. }
$$

We can also note that $r_{2 *}$ satisfies

$$
\frac{\mathrm{d} \bar{T}}{\mathrm{~d} r}\left(r=r_{2 *}\right)=-\frac{r_{0} r_{1}}{r_{1}-r_{0}} \frac{1}{r_{2 *}^{2}}=-\frac{1-T_{2}}{r_{2}-r_{0}} .
$$

Here, in rewriting from the second to the third terms in the right-hand side, we used

$$
1-T_{2}=1-\bar{T}\left(r=r_{2}\right)=1-\frac{r_{0} r_{1}}{r_{1}-r_{0}}\left(\frac{1}{r_{2}}-\frac{1}{r_{1}}\right)=\frac{r_{0} r_{1}}{r_{1}-r_{0}} \frac{r_{2}-r_{0}}{r_{2 *}^{2}} .
$$

In the following, we will use $\gamma_{2} \equiv r_{0} / r_{2}$ (note that $\gamma \leq \gamma_{2}<1$ ) and the expression of $T_{2 *}$ given by

$$
T_{2 *}=\frac{r_{0} r_{1}}{r_{1}-r_{0}}\left(\frac{1}{r_{2 *}}-\frac{1}{r_{1}}\right)=\frac{r_{0} r_{1}}{r_{1}-r_{0}}\left(\frac{1}{\sqrt{r_{0} r_{2}}}-\frac{1}{r_{1}}\right)=\frac{\sqrt{\gamma_{2}}-\gamma}{1-\gamma} .
$$

Let us now consider the ratio of the Rayleigh number in the basal sublayer to that of the entire layer given by

$$
\begin{aligned}
\frac{R a_{2 *}}{R a_{*}} & =\left(1-T_{2}\right)\left(\frac{r_{2}-r_{0}}{r_{1}-r_{0}}\right)^{3} \frac{\exp \left(-E T_{*}\right)}{\exp \left(-E T_{2 *}\right)} \\
& =\frac{\gamma^{3}}{(1-\gamma)^{4}} \frac{\left(1-\gamma_{2}\right)^{4}}{\gamma_{2}{ }^{3}} \exp \left[E\left(T_{2 *}-T_{*}\right)\right] .
\end{aligned}
$$

Differentiating (30) by $\gamma_{2}$ gives

$$
\begin{aligned}
\frac{\partial}{\partial \gamma_{2}}\left(\frac{R a_{2 *}}{R a_{*}}\right) & =\frac{\gamma^{3}}{(1-\gamma)^{4}} \frac{\partial}{\partial \gamma_{2}}\left\{\frac{\left(1-\gamma_{2}\right)^{4}}{\gamma_{2}{ }^{3}} \exp \left[E\left(T_{2 *}-T_{*}\right)\right]\right\} \\
& =\frac{R a_{2 *}}{R a_{*}}\left[-\frac{3+\gamma_{2}}{\gamma_{2}\left(1-\gamma_{2}\right)}+E \frac{\partial T_{2 *}}{\partial \gamma_{2}}\right] \\
& =\frac{R a_{2 *}}{R a_{*}}\left[-\frac{3+\gamma_{2}}{\gamma_{2}\left(1-\gamma_{2}\right)}+E \frac{1}{2(1-\gamma)} \frac{1}{\sqrt{\gamma_{2}}}\right] .
\end{aligned}
$$




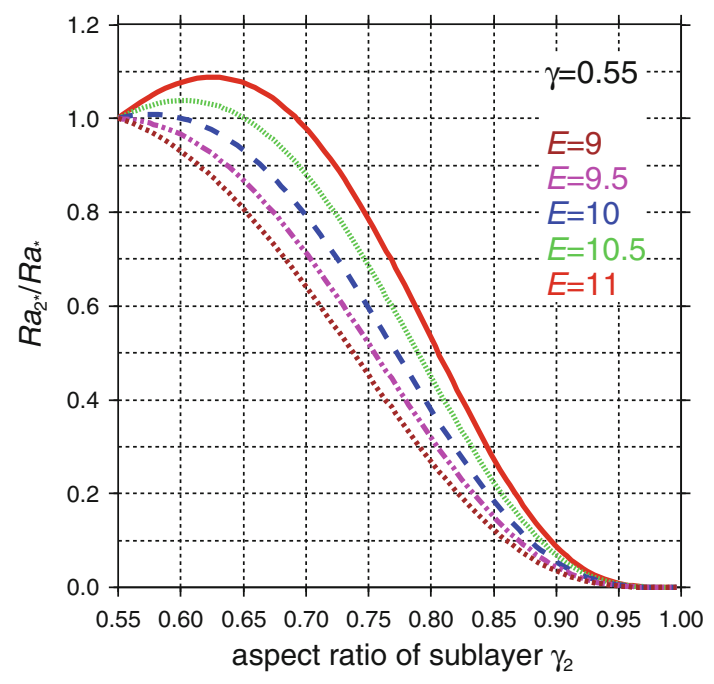

Fig. 11 The plots of the ratio of the local Rayleigh number of the sublayer $R a_{2 *}$ to $R a_{*}$ against the aspect ratio of the sublayer $\gamma_{2}$ obtained for a spherical shell of $\gamma=0.55$ for various values of $E$ indicated in the figure

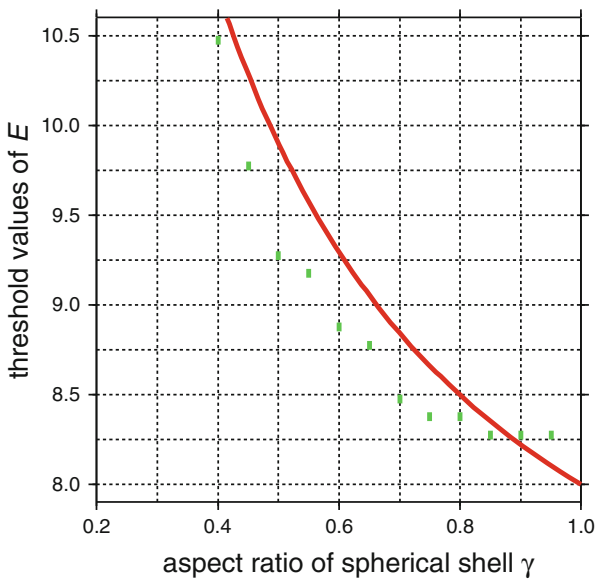

Fig. 12 The variations against the aspect ratio of the spherical shell $\gamma$ of the values of $E_{c}$ for the transition into the stagnant-lid regimes given by (33). Also shown by thick dots are the values of $E_{c}$ by an empirical estimate described in Sect. 3.2.1

This means that the value of $R a_{2 *} / R a_{*}$ is maximum when

$$
\frac{3+\gamma_{2}}{\gamma_{2}\left(1-\gamma_{2}\right)}=E \frac{1}{2(1-\gamma)} \frac{1}{\sqrt{\gamma_{2}}}
$$

In Fig. 11, we plotted the values of $R a_{2 *} / R a_{*}$ given by (30) against the aspect ratio of the sublayer $\gamma_{2}$ for various values of $E$. As can be seen from the figure, for sufficiently large $E$, the plots of $R a_{2 *} / R a_{*}$ against $\gamma_{2}$ have local maxima within the range of $\gamma \leq \gamma_{2}<1$ and, in other words, there exists a sublayer of aspect ratio $\gamma_{2}$ where $R a_{2 *}$ becomes larger than $R a_{*}$. By further assuming that the transition into the ST regime occurs at $E \equiv E_{c}$ which gives $\gamma_{2}=\gamma$, we obtain the relations between $\gamma$ and $E_{c}$ as

$$
E_{c}=\frac{2(3+\gamma)}{\sqrt{\gamma}} .
$$

In order to verify our analytical estimate, we plotted in Fig. 12, the values of $E_{c}$ for the transition into the ST-mode of convection for various values of the aspect ratio of spherical shell $\gamma$ given by (33), together with those derived empirically in Sect. 3.2.1. From the comparison of $E_{c}$, we obtained a satisfactory agreement between the empirical and analytical estimates. 
(a) $R a_{* c 0}$ and $R a_{2 * c 0}$ for $\gamma=0.95$

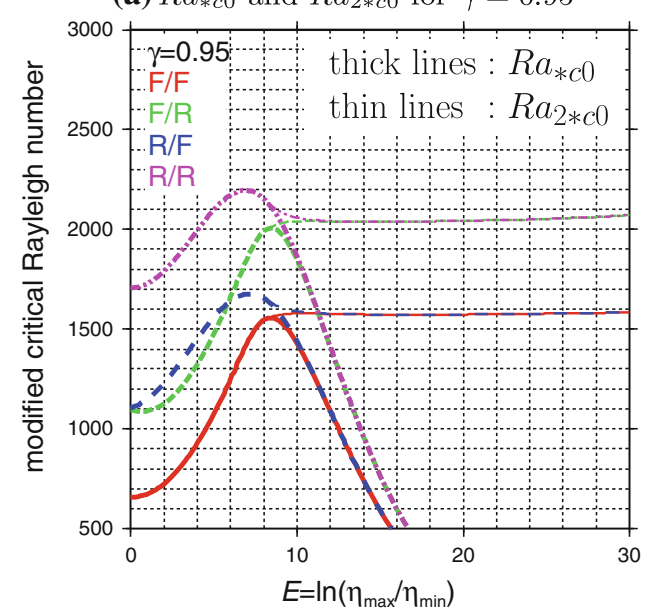

(b) $R a_{* c 0}$ and $R a_{2 * c 0}$ for $\gamma=0.55$

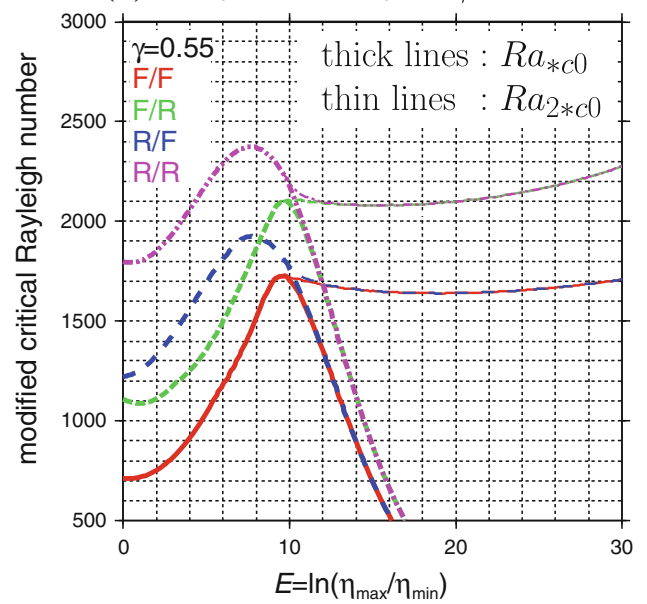

Fig. 13 The plots against $E$ of the absolute minimum values of critical "modified" Rayleigh numbers $R a_{* c 0}$ and $R a_{2 * c 0}$ obtained for the cases with a thin $(\gamma=0.95)$ and $\mathbf{b}$ thick $(\gamma=0.55)$ spherical shells. Plotted by thick lines are $R a_{* c 0}$ of the entire layer, while by thin lines are $R a_{2 * c 0}$ local to the sublayer for the ST convection. The meanings of the labels for the boundary conditions are the same as in Fig. 2

We show in Fig. 13, the plots against $E$ for the cases with $\gamma=0.95$ and $\gamma=0.55$ of the absolute minimum of critical "modified" Rayleigh number $R a_{* c 0}$ calculated by (23), together with those of $R a_{2 * c 0}$ for the sublayer of $\gamma_{2}=\gamma_{2 c 0}$ which satisfies (32) for the ranges of $E$ in the ST regime. The plots of $R a_{* c 0}$ clearly show that the values for both $\gamma$ have maxima at the values of $E$ which correspond to the transition into the ST regime ( $E \simeq 8.3$ for $\gamma=0.95, E \simeq 9.2$ for $\gamma=0.55$ ). In addition, the plots of $R a_{2 * c 0}$ show that in the ST regime, the values of $R a_{2 * c 0}$ are always larger than those of $R a_{* c 0}$. This is another evidence for the validity of our analytical estimate regardless of the aspect ratio $\gamma$ of the spherical shell. We also note that, on the other hand,

(a)

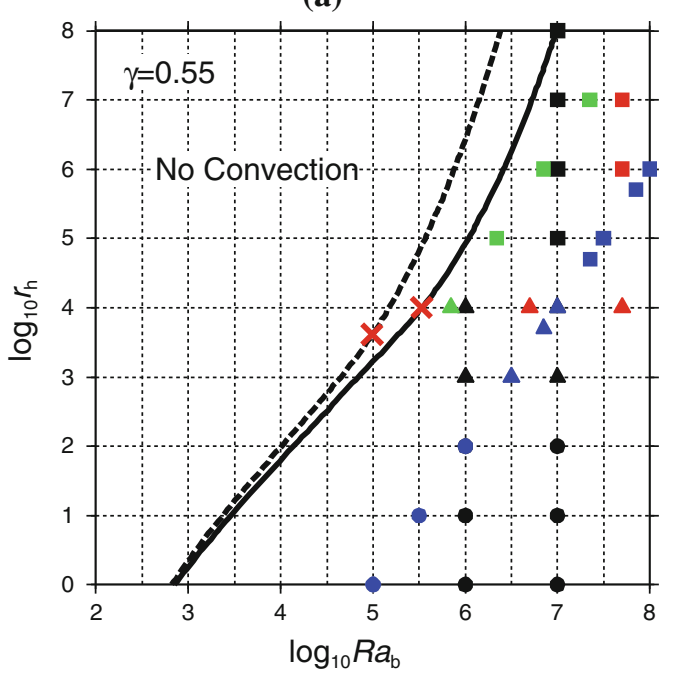

(b)

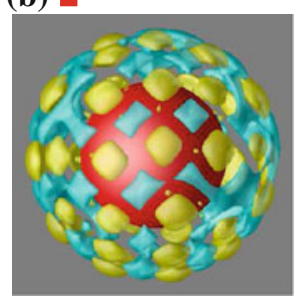

(c) $\Delta$ ("Sluggish-Lid")

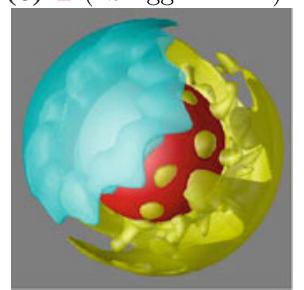

Fig. 14 a A regime diagram of convective flow patterns in the spherical shell with $\gamma=0.55$ in the plane of $R a_{\mathrm{b}}$ (Rayleigh number defined with the viscosity for $T=T_{b}$ ) versus $r_{\eta} \equiv \exp (E)$. Circles, triangles, and squares indicate that convective patterns with narrow, wide, and again narrow cells have been reported in earlier studies with increasing $r_{\eta}$, respectively; black symbols from [28], blue symbols from [18], green symbols from [31], and red symbols from [2]. The thick solid and broken lines show the locations of the threshold for the onset convection obtained in this study for the planar and spherical models, respectively. The red crosses in the figure indicate the locations of the regime boundaries between the ST and other modes on the thick lines. $\mathbf{b}$ and $\mathbf{c}$ Examples of convective flow patterns obtained by [2] for $\mathbf{b} R a_{\mathrm{b}}=5 \times 10^{7}$ and $r_{\eta}=10^{6}$ and $\mathbf{c} R a_{\mathrm{b}}=5 \times 10^{7}$ and $r_{\eta}=10^{4}$. Shown are the three-dimensional plots of the distributions of lateral thermal anomaly $\delta T \equiv T-\langle T\rangle$, where $\langle T\rangle$ is the horizontally averaged temperature at given $r$. Indicated in cyan are the cold thermal anomalies with $\delta T \leq-0.1$, while in yellow are the hot anomalies with $\delta T \geq 0.1$. Red spheres show the inner bottom boundary of the convecting layer 
there is a slight difference between the cases with $\gamma=0.95$ and 0.55 in the variations of $R a_{2 * c 0}$ for very large $E$. For a thin spherical shell $(\gamma=0.95)$, the values $R a_{2 * c 0}$ are almost constant for $E$ up to 30, as in the case within a planar layer (see Sect. 3.1). For $\gamma=0.55$, in contrast, the values of $R a_{2 * c 0}$ moderately vary depending on $E$ with a gradual increase for large $E$, since the aspect ratio $\gamma_{2 c 0}$ of the sublayer significantly increases with increasing $E$ for a thick spherical shell.

\section{Discussion and concluding remarks}

We performed a linear stability analysis for the onset of thermal convection of a fluid with strongly temperature-dependent viscosity in a basally heated planar layer or a spherical shell. In this study, we focused on the condition for the stagnant-lid (ST) mode of convection with increasing the temperature dependence of viscosity, with particular emphasis on the changes in vertical structures of incipient flows. We first developed an empirical but quantitative criterion for the dominance of the ST convection, based on the well-established results for a planar layer. The criterion comes from an extension of the idea of [19], which postulates that in the ST regime, the onset of convection occurs only in a sublayer that does not extend to the upper boundary. We proposed a quantity $\Delta_{h}$, defined by (21) or (22) depending on the model geometries, which represents the extent of conversion between horizontal and vertical flows at a particular height and, in other words, the source regions of ascending and descending flows. From a series of calculations varying the model geometries as well as the values of parameter $E$ describing the temperature-dependent viscosity, we found that the dominance of the ST convection is significantly affected by the model geometries, such as the aspect ratio of spherical shell $\gamma$. The dominance occurs at $E \simeq 8$ in a thin spherical shell (large $\gamma$ ), as in the case with a planar layer, while it occurs at larger $E$ in a thick spherical shell (small $\gamma$ ). Our findings indicate the unsoundness of the classification of the convecting regimes in a thick spherical shell relevant to the Earth's mantle $(\gamma \simeq 0.55)$, which has been simply based on the results obtained in a planar layer.

We also developed an analytical estimate for the transition into the ST regimes in a spherical shell, following the idea of [19] where convection is assumed to begin in a sublayer with a maximum Rayleigh number. Our analysis showed that the fact that the values of $E$ for the dominance of the ST regimes depend on the aspect ratio $\gamma$ can be explained by a slight modification of the definition of the Rayleigh number from that employed for a planar layer. As has been demonstrated in Sect. 3.2.2, it is quite important to appropriately choose the values of "representative" viscosity and, in other words, the "representative" temperature $T_{*}$ or the position $r_{*}$ of the fluid of strongly temperature-dependent viscosity in a spherical shell. This is in a stark contrast to the case in a planar layer, where the values of $T_{*}$ and $r_{*}$ are naturally chosen to be those at the mid-depth of the layer $\left(T_{*}=\frac{1}{2}\right.$ and $r_{*}=\frac{1}{2}$ ). Indeed, as can be seen in Fig. 10a, the values of $r_{*}$ significantly deviate from that for the mid-depth of the spherical shell $\left(=\frac{1}{2}\left(r_{0}+r_{1}\right)\right)$ with decreasing $\gamma$. In addition, for $\gamma<1$, the values of $T_{*}$ are different from either the mean of the top and bottom values $(=0.5)$ or the volumetric average $T_{\mathrm{av}}$ in the entire layer. This indicates that an appropriate choice of the Rayleigh number is of crucial importance for understanding the convecting regimes in a thick spherical shell.

We will apply our results to a classification of the convecting regimes in a thick spherical shell relevant to the Earth's mantle. We show with the thick solid line in Fig. 14a the variation of the absolute minimum of the critical Rayleigh number against the temperature dependence of viscosity obtained for a spherical shell of $\gamma=0.55$. In the figure, we plotted, instead of $E$ and $R a$, the viscosity contrast across the layer $r_{\eta} \equiv \exp (E)$ and the Rayleigh number defined with the viscosity at the bottom surface $R a_{\mathrm{b}} \equiv \exp (E / 2) R a$, for a direct comparison with the earlier studies $[3,9,10,15]$. We also show by the dashed line the relation with $R a_{\mathrm{b}}$ and $r_{\eta}$ for a planar layer. In addition, the red crosses indicate the transitions into the ST regimes on the curves. As can be seen from the figure, the curves of $r_{\eta}$ against $R a_{\mathrm{b}}$ have slight bends near the red crosses, regardless of the model geometries. This is also an evidence for the significance of the criterion for the transition into the ST regimes proposed in this study.

We also show in Fig. 14a, the classification of convective regimes, inferred from the changes in the horizontal length scales of convection cells, obtained by earlier numerical experiments of thermal convection in three-dimensional spherical shells $[2,13,18,28,31]$. Among the symbols in the figure, the triangles indicate the conditions for a so-called "sluggish-lid" (SL) mode of convection which is characterized by convection cells of large horizontal scales (see Fig. 14c), while the squares indicate those for a convection with small horizontal scales (see Fig. 14b). In particular, the mode of convection denoted by the squares in the figure has been considered to be identical to the ST mode in most of the earlier studies [13,20,24,28], although it has been reported from two-dimensional convection models with a moderately wide box [3] that the changes from wide to narrow convection cells with increasing $r_{\eta}$ do not necessarily coincide with that in vertical flow structures. 
The results from our linear stability analysis suggest that the conclusion drawn from two-dimensional Cartesian models [3] is most likely to be true for the model with spherical shell geometry relevant to the Earth's mantle $(\gamma=0.55)$. Indeed, as can be seen in Fig. 14a, the changes in the vertical structures of incipient flows occur at around $r_{\eta} \simeq 10^{4}$ (i.e., $E \simeq 9.2$ ), while, from earlier numerical studies [2,28,31], that the collapse of the SL-mode of convection occurs at the values of $r_{\eta}$ larger than $10^{4}$. It can, therefore, be conjectured that, for a range of $10^{4} \lesssim r_{\eta} \lesssim 10^{4.5}$, there exists an ST-mode of convection with convection cells of large horizontal length scales. Of course, the present findings are not entirely applicable to the classification of convecting regimes from finite-amplitude flows, since our results are valid only near the critical condition (indicated by the thick lines in Fig. 14a). In addition, the transition into the ST regimes does not necessarily occur at $r_{\eta} \simeq 10^{4}$ when the Rayleigh number is sufficiently higher than the critical value. However, as can be seen from Fig. 14c, the SL-mode of convection obtained with $r_{\eta}=10^{4}$ and the Rayleigh number far larger than the critical value $\left(R a_{\mathrm{b}}=5 \times 10^{7}\right)$ have a feature quite similar to that of "elongated ST-mode" of convection, which has been identified by an earlier numerical model using two-dimensional moderately wide Cartesian geometry [3]. The convective flow pattern of these cases is characterized by convection cells of large aspect ratio superimposed by several minor ascending and descending plumes due to the instability in the top and bottom thermal boundary layers (TBLs). Moreover, the minor descending plumes originating from the base of the cold TBL do not break the wide convection cells into narrow ones, implying that the cold TBL is stiff enough to prevent the instability from penetrating upward into the TBL. The similarity of "SL-mode" and "elongated ST-mode" suggest that our conjecture is most likely to be valid for, at least, around $r_{\eta}=10^{4}$ even when the Rayleigh number is sufficiently large.

One of the most important inferences from this study is, we believe, that much more careful studies are needed for the transitions in convective flow patterns in a spherical shell geometry. This is largely because it is of crucial importance for understanding the convective planforms in the mantle of the terrestrial planets. It has been commonly believed that the convective motion in the mantle of terrestrial planets is characterized by (i) thick stiff lids along the top cold surfaces and (ii) convection cells of large horizontal scales $[6,14,17,25,30$, for example]. However, it has also be considered that a mode of thermal convection can be hardly obtained solely from the effect of temperature-dependent viscosity, which bears these two features simultaneously. For instance, when the viscosity is strongly temperature dependent, convection cells are thought to have small horizontal scales beneath highly viscous and stiff lids. On the other hand, for a convection with cells of large horizontal scales, the cold TBLs are not viscous enough to act as a stiff lid. In order to simultaneously reproduce these two features, various agents, other than temperature-dependent viscosity, have been incorporated into the numerical models of mantle convection, such as the effects of depth-dependent viscosity [28], internal heating [8], adiabatic compression [23], and yielding within the cold TBLs [21,22,27]. Contrary to earlier conjectures described above, the present results indicate that the ST-mode of convection with elongated convection cells is most likely to occur, under certain conditions, solely from the temperature-dependent viscosity. Certainly, such agents, if they operate together with the temperature-dependent viscosity, would play an important role in simultaneously reproducing convective patterns with wide convection cells beneath cold and stiff lids. It may be an oversimplified view, however, that such agents are inevitable mechanisms in mantle convection of terrestrial planets, since the effects of temperature-dependent viscosity have not been fully resolved on the changes in vertical flow structures. We, thus, conclude that it is still an important issue to carefully study the effects of temperature-dependent viscosity on the convective planforms, both vertically and horizontally and with a three-dimensional spherical shell geometry, in order to deepen the insights into the nature of mantle convection of terrestrial planets.

Acknowledgments We thank Drs. Takashi Nakagawa, Shijie Zhong, and David A. Yuen for fruitful discussion. We also thank Sabrina Whitaker for correcting the grammatical and typological errors in the manuscript. We thank three anonymous reviewers for valuable comments which greatly improved the manuscript. We greatly acknowledge thorough support from the Global COE program from the Ministry of Education, Culture, Sports, and Technology (MEXT) of Japan. MK acknowledges financial supports from Grant-in-Aid for Scientific Research (C; \#19540452) and (B; \#22340127) from Japan Society for the Promotion of Science.

Open Access This article is distributed under the terms of the Creative Commons Attribution Noncommercial License which permits any noncommercial use, distribution, and reproduction in any medium, provided the original author(s) and source are credited.

\section{A The details of numerical techniques}

In this study, the critical Rayleigh number $R a_{c}$ for a given condition (i.e., boundary conditions, the values of $E$ and $K$ ) is numerically calculated in two steps. First, for a given Rayleigh number $R a$, we calculated the 
infinitesimal perturbation which yields the largest growth rate $a_{\max }$. We then sought for the value of $R a_{c}$ which gives $a_{\max }=0$, by iteratively varying $R a$. In this section, we will describe the details of the each technique in turn. In the following, for simplicity, we will write the eigenequation to be solved as

$$
\boldsymbol{A x}=\lambda \boldsymbol{x} .
$$

Here the matrix $\boldsymbol{A}$ represents the spatial discretization, and the eigenvalue $\lambda$ and eigenvector $\boldsymbol{x}$ stand for the growth rate and infinitesimal perturbations of temperature and velocity, respectively.

\section{A.1 Solution of the largest eigenvalue $\lambda_{\max }$}

The numerical difficulty in solution of the largest eigenvalue $\lambda_{\max }$ largely comes from the fact that $\lambda_{\max }$ can be either positive or negative depending on the conditions to be considered. This implies that basic numerical techniques (such as the power method) which seek for the dominant eigenvalue (i.e., the one with the largest absolute value) cannot be directly used. However, as will be shown below, a slight modification of the original eigenequations enables us to use the power method in order to obtain $\lambda_{\max }$.

In the present numerical algorithm, we seek for the eigenvalues $\tilde{\lambda}$ of an "auxiliary" matrix $\tilde{A}$ given by

$$
\tilde{\boldsymbol{A}}=\boldsymbol{I}+\Delta t \boldsymbol{A},
$$

instead of directly solving for the eigenvalues $\lambda$ of $\boldsymbol{A}$ in (34). Here $\boldsymbol{I}$ is an identity matrix and $\Delta t$ is a small positive constant similar to a time increment. By multiplying an eigenvector $\boldsymbol{x}$ of $\boldsymbol{A}$ with (35), we get

$$
\tilde{\boldsymbol{A}} \boldsymbol{x}=\boldsymbol{x}+\Delta t \boldsymbol{A} \boldsymbol{x}=(1+\lambda \Delta t) \boldsymbol{x} .
$$

This means that both $\boldsymbol{A}$ and $\tilde{\boldsymbol{A}}$ have the same set of eigenvectors and that the following relation holds between the eigenvalues $\lambda$ and $\tilde{\lambda}$ of the two matrices:

$$
\tilde{\lambda}=1+\lambda \Delta t .
$$

In addition, let us assume that the parameter $\Delta t$ is taken to be small enough to satisfy

$$
\Delta t<\frac{1}{|\lambda \min |}
$$

where $\lambda_{\min }$ is the smallest eigenvalue of $\boldsymbol{A}$. Together with (37), this leads to

$$
\tilde{\lambda} \geq 1+\lambda_{\min } \Delta t>1+\frac{\lambda_{\min }}{\left|\lambda_{\min }\right|}=0 .
$$

Namely, all of the eigenvalues $\tilde{\lambda}$ of $\tilde{\boldsymbol{A}}$ are positive. This means that the largest eigenvalue $\tilde{\lambda}_{\text {max }}$ can be easily solved for by, for example, the power method since $\tilde{\lambda}_{\max }=\left|\tilde{\lambda}_{\max }\right|$. In other words, the eigenvalue $\lambda_{\max }$ of the matrix $\boldsymbol{A}$ can be derived from $\tilde{\lambda}_{\max }$ of the matrix $\tilde{\boldsymbol{A}}$ via

$$
\lambda_{\max }=\frac{\tilde{\lambda}_{\max }-1}{\Delta t},
$$

regardless of the sign of $\lambda_{\max }$.

We finally consider an appropriate choice of the positive parameter $\Delta t$. Suppose a following "auxiliary" evolutionary equation

$$
\frac{\mathrm{d} \boldsymbol{y}}{\mathrm{d} t}=\boldsymbol{A y} .
$$

By discretizing the above equation in the direction of $t$ by a first-order explicit scheme, we get

$$
\frac{\boldsymbol{y}^{n+1}-\boldsymbol{y}^{n}}{\Delta t}=\boldsymbol{A} \boldsymbol{y}^{n} .
$$

By using (35), this equation can be rewritten as

$$
\boldsymbol{y}^{n+1}=(\boldsymbol{I}+\Delta t \boldsymbol{A}) \boldsymbol{y}^{n}=\tilde{A} \boldsymbol{y}^{n} .
$$

This means that the multiplication of $\tilde{\boldsymbol{A}}$ is just a time-marching calculation of the (41) by a first-order explicit scheme. In other words, the parameter $\Delta t$ must be chosen to satisfy the Courant-Friedrichs-Lewy (CFL) condition of (41). In the present study, the values of $\Delta t$ are determined from the CFL condition of (4). 
A.2 Solution of the critical Rayleigh number

By using the technique in the previous subsection, we can obtain the value of $a_{\max }$ for a given Rayleigh number $R a$. Next, we seek for the value of the critical Rayleigh number $R a_{c}$, which yields $a_{\max }=0$, by the well-known bisection method. The bisection method is a root-finding method that repeatedly bisects an interval and then selects a subinterval where a root must lie for further processing. Owing to the monotonous relation between $R a$ and $a_{\max }$, the bisection method always converges to the correct $R a_{c}$.

\section{References}

1. Chandrasekhar, S.: Hydrodynamic and Hydromagnetic Stability. The Clarendon Press, Oxford (1961)

2. Kameyama, M., Kageyama, A., Sato, T.: Multigrid-based simulation code for mantle convection in spherical shell using Yin-Yang grid. Phys. Earth Planet. Inter. 171(1-4), 19-32 (2008). doi:10.1016/j.pepi.2008.06.025

3. Kameyama, M., Ogawa, M.: Transitions in thermal convection with strongly temperature-dependent viscosity in a wide box. Earth Planet. Sci. Lett. 180(3-4), 355-367 (2000). doi:10.1016/S0012-821X(00)00171-0

4. Karato, S.I., Wu, P.: Rheology of the upper mantle: a synthesis. Science 260(5109), 771-778 (1993). doi:10.1126/science. 260.5109 .771

5. Kiefer, W.S., Li, Q.: Mantle convection controls the observed lateral variations in lithospheric thickness on present-day Mars. Geophys. Res. Lett. 36, L18203 (2009). doi:10.1029/2009GL039827

6. Konopliv, A.S., Banerdt, W.B., Sjogren, W.L.: Venus gravity: 180th degree and order model. Icarus 139(1), 3-18 (1999). doi:10.1006/icar.1999.6086

7. Li, Q., Kiefer, W.S.: Mantle convection and magma production on present-day Mars: Effects of temperature-dependent rheology. Geophys. Res. Lett. 34, L16203 (2007). doi:10.1029/2007GL030544

8. McNamara, A.K., Zhong, S.: Degree-one mantle convection: dependence on internal heating and temperature-dependent rheology. Geophys. Res. Lett. 32, L01301 (2005). doi:10.1029/2004GL021082

9. Moresi, L.N., Solomatov, V.: Numerical investigation of 2D convection with extremely large viscosity variations. Phys. Fluids 7, 2154-2162 (1995)

10. Ogawa, M., Schubert, G., Zebib, A.: Numerical simulations of three-dimensional thermal convection in a fluid with strongly temperature-dependent viscosity. J. Fluid Mech. 233, 299-328 (1991)

11. Ratcliff, J.T., Schubert, G., Zebib, A.: Effects of temperature-dependent viscosity on thermal convection in a spherical shell. Physica D Nonlinear Phenomena 97(1-3), 242-252 (1996). doi:10.1016/0167-2789(96)00150-9

12. Ratcliff, J.T., Schubert, G., Zebib, A.: Steady tetrahedral and cubic patterns of spherical shell convection with temperaturedependent viscosity. J. Geophys. Res. 101(B11), 25,473-25,484 (1996). doi:10.1029/96JB02097

13. Ratcliff, J.T., Tackley, P.J., Schubert, G., Zebib, A.: Transitions in thermal convection with strongly variable viscosity. Phys. Earth Planet. Inter. 102(3-4), 201-212 (1997). doi:10.1016/S0031-9201(97)00013-7

14. Schubert, G., Turcotte, D.L., Olson, P.: Mantle Convection in the Earth and Planets. Cambridge University Press, Cambridge (2001)

15. Solomatov, V.S.: Scaling of temperature- and stress-dependent viscosity convection. Phys. Fluids 7, 266-274 (1995)

16. Solomatov, V.S., Moresi, L.N.: Three regimes of mantle convection with non-Newtonian viscosity and stagnant-lid convection on the terrestrial planets. Geophys. Res. Lett. 24(15), 1907-1910 (1997). doi:10.1029/97GL01682

17. Šrámek, O., Zhong, S.: Long-wavelength stagnant-lid convection with hemispheric variation in lithospheric thickness: link between Martian crustal dichotomy and Tharsis?. J. Geophys. Res. 115, E09010 (2010). doi:10.1029/2010JE003597

18. Stemmer, K., Harder, H., Hansen, U.: A new method to simulate convection with strongly temperature- and pressure-dependent viscosity in a spherical shell: applications to the Earth's mantle. Phys. Earth Planet. Inter. 157(3-4), 223-249 (2006). doi:10.1016/j.pepi.2006.04.007

19. Stengel, K.C., Oliver, D.S., Booker, J.R.: Onset of convection in a variable-viscosity fluid. J. Fluid Mech. 120, 411431 (1982). doi:10.1017/S0022112082002821

20. Tackley, P.J.: Effects of strongly variable viscosity on three-dimensional compressible convection in planetary mantles. J. Geophys. Res. 101(B2), 3311-3332 (1996)

21. Tackley, P.J.: Self-consistent generation of tectonic plates in time-dependent, three-dimensional mantle convection simulations 1. Pseudoplastic yielding. Geochem. Geophys. Geosyst. 1(8), 1021 (2000). doi:10.1029/2000GC000036

22. Tackley, P.J.: Self-consistent generation of tectonic plates in time-dependent, three-dimensional mantle convection simulations 2. Strain weakening and asthenosphere. Geochem. Geophys. Geosyst. 1(8), 1026 (2000). doi:10.1029/2000GC000043

23. Tackley, P.J., Xie, S., Nakagawa, T., Hernlund, J.W.: Experimental studies of mantle convection: philosophy, accomplishments and thermo-chemical structure and evolution. In: van der Hilst, R.D., Bass, J., Matas, J., Trampert, J. (eds.) Earth's Deep Mantle: Structure, Composition, and Evolution, Geophysical Monograph, vol. 160, pp. 83-99. AGU (2005)

24. Trompert, R., Hansen, U.: On the Rayleigh number dependence of convection with a strongly temperature-dependent viscosity. Phys. Fluids 10(2), 351-364 (1998). doi:10.1063/1.869527

25. Turcotte, D.L., Schubert, G.: Geodynamics. pp. 456 2nd edn. Cambridge University Press, Cambridge (2002)

26. Weertman, J.: The creep strength of the Earth's mantle. Rev. Geophys. 8, 145-168 (1970)

27. Yoshida, M.: Mantle convection with longest-wavelength thermal heterogeneity in a 3-D spherical model: degree one or two?. Geophys. Res. Lett. 35, L23302 (2008). doi:10.1029/2008GL036059

28. Yoshida, M., Kageyama, A.: Low-degree mantle convection with strongly temperature- and depth-dependent viscosity in a three-dimensional spherical shell. J. Geophys. Res. 111, B03412 (2006). doi:10.1029/2005JB003905

29. Zebib, A.: Linear and weakly nonlinear variable viscosity convection in spherical shells. Theor. Comput. Fluid Dyn. 4(5), 241-253 (1993). doi:10.1007/BF00417930 
30. Zhao, D.: Global tomographic images of mantle plumes and subducting slabs: insight into deep Earth dynamics. Phys. Earth Planet. Inter. 146(1-2), 3-34 (2004). doi:10.1016/j.pepi.2003.07.032

31. Zhong, S., McNamara, A., Tan, E., Moresi, L., Gurnis, M.: A benchmark study on mantle convection in a 3-D spherical shell using CitcomS. Geochem. Geophys. Geosyst. 9, Q10017 (2008). doi:10.1029/2008GC002048 